ARTICLE

https://doi.org/10.1038/s41467-019-10839-0

\title{
Metformin induces lipogenic differentiation in myofibroblasts to reverse lung fibrosis
}

\author{
Vahid Kheirollahi ${ }^{1,2}$, Roxana M. Wasnick (10 2, Valentina Biasin ${ }^{3}$, Ana Ivonne Vazquez-Armendariz ${ }^{2}$, \\ Xuran Chu ${ }^{2,4}$, Alena Moiseenko², Astrid Weiss², Jochen Wilhelm², Jin-San Zhang ${ }^{1}{ }^{4}$, Grazyna Kwapiszewska ${ }^{3}$, \\ Susanne Herold ${ }^{2}$, Ralph T. Schermuly (10 ${ }^{2}$, Bernard Mari ${ }^{5,6}$, Xiaokun Li ${ }^{4}$, Werner Seeger (iD ${ }^{2}$, \\ Andreas Günther (1D 2, Saverio Bellusci, ${ }^{1,2,4}$ \& Elie El Agha(iD ${ }^{1,2,4}$
}

Idiopathic pulmonary fibrosis (IPF) is a fatal disease in which the intricate alveolar network of the lung is progressively replaced by fibrotic scars. Myofibroblasts are the effector cells that excessively deposit extracellular matrix proteins thus compromising lung structure and function. Emerging literature suggests a correlation between fibrosis and metabolic alterations in IPF. In this study, we show that the first-line antidiabetic drug metformin exerts potent antifibrotic effects in the lung by modulating metabolic pathways, inhibiting TGF $\beta 1$ action, suppressing collagen formation, activating PPAR $\gamma$ signaling and inducing lipogenic differentiation in lung fibroblasts derived from IPF patients. Using genetic lineage tracing in a murine model of lung fibrosis, we show that metformin alters the fate of myofibroblasts and accelerates fibrosis resolution by inducing myofibroblast-to-lipofibroblast transdifferentiation. Detailed pathway analysis revealed a two-arm mechanism by which metformin accelerates fibrosis resolution. Our data report an antifibrotic role for metformin in the lung, thus warranting further therapeutic evaluation.

\footnotetext{
${ }^{1}$ Key Laboratory of Interventional Pulmonology of Zhejiang Province, Department of Pulmonary and Critical Care Medicine, The First Affiliated Hospital of Wenzhou Medical University, 325035 Wenzhou, China. ${ }^{2}$ Cardio-Pulmonary Institute (CPI) and Department of Pulmonary and Critical Care Medicine and Infectious Diseases, Universities of Giessen and Marburg Lung Center (UGMLC), Member of the German Center for Lung Research (DZL), Justus-Liebig University Giessen, 35392 Giessen, Germany. ${ }^{3}$ Ludwig Boltzmann Institute for Lung Vascular Research, Otto Loewi Research Center, Medical University Graz, 8010 Graz, Austria. ${ }^{4}$ International Collaborative Center on Growth Factor Research and School of Pharmaceutical Sciences, Wenzhou Medical University, 325035 Wenzhou, China. ${ }^{5}$ Institut de Pharmacologie Moleculaire et Cellulaire (IPMC), Centre National de la Recherche Scientifique (CNRS), UMR 7275, Sophia Antipolis 06560 Valbonne, France. ${ }^{6}$ Universite Cote d'Azur, 06108 Nice, France. Correspondence and requests for materials should be addressed to S.B. (email: saverio.bellusci@innere.med.uni-giessen.de) or to E.E.A. (email: elie.el-agha@innere.med.uni-giessen.de)
} 
diopathic pulmonary fibrosis (IPF) is a fatal lung disease of unknown etiology. This disease is more common among the elderly and the average survival rate following diagnosis is only 2-3 years ${ }^{1}$. Histopathological examination of IPF lungs typically reveals extensive alveolar scarring; i.e., replacement of normal alveoli by fibrous scars containing myofibroblasts. The latter cells are considered to be the main source of excessive extracellular matrix (ECM) protein deposition, particularly collagen ${ }^{2}$, not only in IPF lungs but also in fibrosis of other organs. Due to its progressive nature and since the process of scar formation is part of natural wound healing, IPF is widely regarded as an aberrant wound healing response to repetitive epithelial injury ${ }^{3}$.

The cellular source of myofibroblasts has been a subject of debate in recent years. The paradigm is that identifying the precursor cell of the myofibroblast might pave the way for preventive and/or therapeutic, selective intervention in IPF patients. In this context, it is suggested that the myofibroblast pool is heterogeneous, and derives from multiple sources such as resident fibroblasts, circulating fibrocytes, perivascular mesenchymal cells, and alveolar epithelial cells ${ }^{4-7}$. Using genetic lineage tracing, we have recently identified the resident lipid-droplet-containing interstitial fibroblast, or lipofibroblast, as a precursor cell for the myofibroblast in the bleomycin model of lung fibrosis in mice ${ }^{8}$. We also found that myofibroblasts retain their plasticity and are able to revert to the type 2 alveolar epithelial cell (AT2)-supportive lipofibroblast fate during recovery ${ }^{8}$. We finally showed that peroxisome proliferator-activated receptor gamma (PPAR $\gamma$ ) agonist, rosiglitazone, induces lipogenic differentiation and inhibits transforming growth factor beta 1 (TGF $\beta 1$ )-induced myogenic differentiation in primary human lung fibroblasts ${ }^{8}$.

The therapeutic effect of rosiglitazone in the murine model of bleomycin-induced pulmonary fibrosis is well established ${ }^{9,10}$. Rosiglitazone belongs to the thiazolidinedione class of PPAR $\gamma$ agonists and is a potent antidiabetic agent. Ongoing research regarding its role in suppressing inflammation ${ }^{11}$, inducing apoptosis and suppressing tumor growth ${ }^{12}$ in parallel to inhibiting TGF $\beta 1$ signaling 8 , makes it a good therapeutic candidate for IPF patients. Since rosiglitazone might not be the ideal therapeutic option for patients who are at risk of developing ischemic heart disease $\mathrm{e}^{13}$, exploring the applicability of alternative therapeutic compounds is clinically relevant.

Interestingly, IPF is associated with metabolic disorders. For example, a recent report has shown that IPF lungs display alterations in several metabolites linked to energy consumption ${ }^{14}$. In addition, there is evidence suggesting that several types of lipid molecules present in blood plasma could be used as biomarkers for IPF $^{15}$. One study has even suggested that type 2 diabetes might be a risk factor for developing IPF ${ }^{16}$. Although it remains unclear whether these metabolic abnormalities represent a cause or consequence of the disease, the link between fibrosis and metabolic alterations in the lung raises the question whether antidiabetic drugs can be good candidates for antifibrotic therapy. Metformin is another antidiabetic drug that has been extensively used to manage diabetic patients. It inhibits gluconeogenesis in the liver and increases peripheral glucose utilization by sensitizing cells to insulin. In fact, metformin and rosiglitazone have been used in combination to treat patients with type 2 diabetes. Both agents decrease the amount of glucose produced by the liver and absorbed by the intestine.

Many studies have reported the therapeutic effects of metformin in non-diabetic diseases such as non-small-cell lung cancer ${ }^{17}$, prostate cancer ${ }^{18}$, and cardiovascular diseases ${ }^{19}$. Moreover, it has been suggested that intraperitoneal administration of metformin attenuates bleomycin-induced lung fibrosis in mice via $\mathrm{NADPH}$ oxidase 4 (NOX4) suppression ${ }^{20}$. A more recent report has shown that metformin accelerates resolution of bleomycin-induced pulmonary fibrosis, suggesting activation of AMP-activated protein kinase (AMPK) as key underlying signaling event, leading to downregulation of alpha smooth muscle actin (ACTA2) and collagen, and increasing myofibroblast autophagy and ECM turnover $^{21}$.

In the current study, we hypothesized that metformin accelerates fibrosis resolution by inducing lipogenic differentiation in lung fibroblasts, while inhibiting TGF $\beta 1$-mediated myogenic differentiation. To test this hypothesis, we used primary cultures of human IPF-derived lung fibroblasts, cultures of precision-cut lung slices (PCLS) derived from human IPF patients, and genetic lineage tracing in the context of the bleomycin model of lung fibrosis in mice. We show that metformin accelerates fibrosis reversal by altering the fate of myofibroblasts in the lung. Mechanistically, metformin induces lipogenic differentiation in myofibroblasts via a mechanism involving bone morphogenetic protein $2(B M P 2)$ upregulation and PPAR $\gamma$ activation, and inhibits TGF $\beta 1$-induced collagen production via AMPK activation. Our data highlight the potential for using metformin to treat IPF patients.

\section{Results}

Metformin induces lipid accumulation in IPF lung fibroblasts. In order to investigate whether metformin impacts lipogenic versus myogenic fibroblastic phenotypes in the lung, we carried out a dose-finding study. Human lung fibroblasts isolated from 7 IPF patients were starved for $24 \mathrm{~h}$ before being treated with 1,5 , or $10 \mathrm{mM}$ metformin for $72 \mathrm{~h}$ (Supplementary Fig. 1). Treatment of cells with $5 \mathrm{mM}$ metformin resulted in significant upregulation of the lipogenic markers adipose differentiation-related protein (a.k.a. perilipin 2, PLIN2, 18.3 folds, Supplementary Fig. 1A) and PPARg (6.6 folds, Supplementary Fig. 1B) in parallel to a significant 9.7-fold downregulation of COL1A1 (Supplementary Fig. 1C). Treatment with $10 \mathrm{mM}$ metformin led to comparable results (Supplementary Fig. 1A-C). No induction of apoptosis was observed in response to metformin treatment $(5 \mathrm{mM})$ (Supplementary Fig. 1D). Therefore, we decided to use the $5 \mathrm{mM}$ concentration for the rest of the study.

In order to gain further insights into the dynamics of this process, human lung fibroblasts isolated from 5 IPF patients were treated with $5 \mathrm{mM}$ metformin and analyzed after 48, 72, and $96 \mathrm{~h}$ (Supplementary Fig. 2A). Analysis at $48 \mathrm{~h}$ showed a significant 3.4-fold downregulation of COL1A1 expression (Supplementary Fig. 2D) but without affecting lipogenic marker expression (Supplementary Fig. 2B, C) or lipid-droplet accumulation (as shown by staining with the neutral lipid dye LipidTOX) (Supplementary Fig. 2K, L, Q). At $72 \mathrm{~h}$, a significant 9.6-fold downregulation of COL1A1 (Supplementary Fig. 2G) was accompanied by significant upregulation of PLIN2 (33.5 folds, Supplementary Fig. 2E) and PPARg (12.4 folds, Supplementary Fig. $2 \mathrm{~F}$ ) and a 4.3-fold increase in lipid-droplet accumulation (Supplementary Fig. 2M, N, R). The effect of metformin was comparable at $96 \mathrm{~h}$ (Supplementary Fig. 2H-J, O, P, S). These data indicate that metformin first leads to COL1A1 downregulation and then induces lipogenic marker expression in primary human IPF lung fibroblasts. The 72-h time point was chosen for subsequent analyses.

Following the establishment of an optimal dose and time point for metformin treatment, pulmonary fibroblasts isolated from $12 \mathrm{IPF}$ patients were treated with $5 \mathrm{mM}$ metformin and analyzed after $72 \mathrm{~h}$ (Fig. 1a). The results showed significant upregulation of PLIN2 (22.1 folds, Fig. 1b) and PPARg (8.3 folds, Fig. 1c), and a robust 8.1-fold downregulation of COL1A1 (Fig. 1d). Despite the variation in the response of fibroblasts isolated from different patients, the pattern of response was highly significant and clearly 

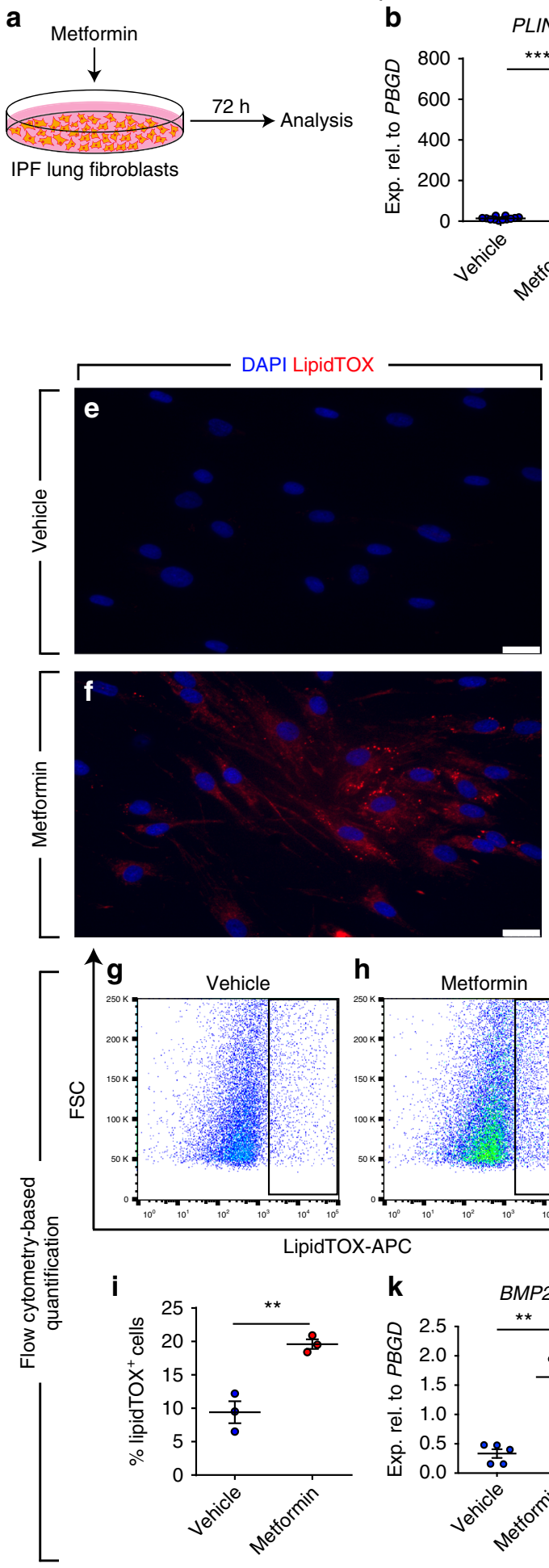
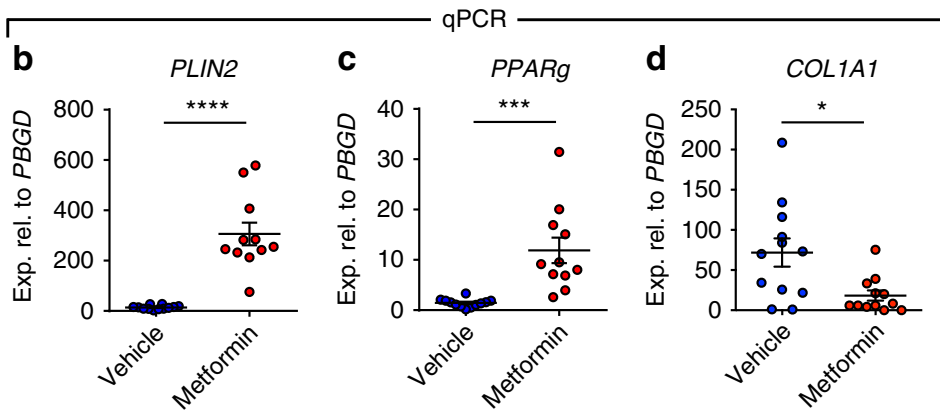

j

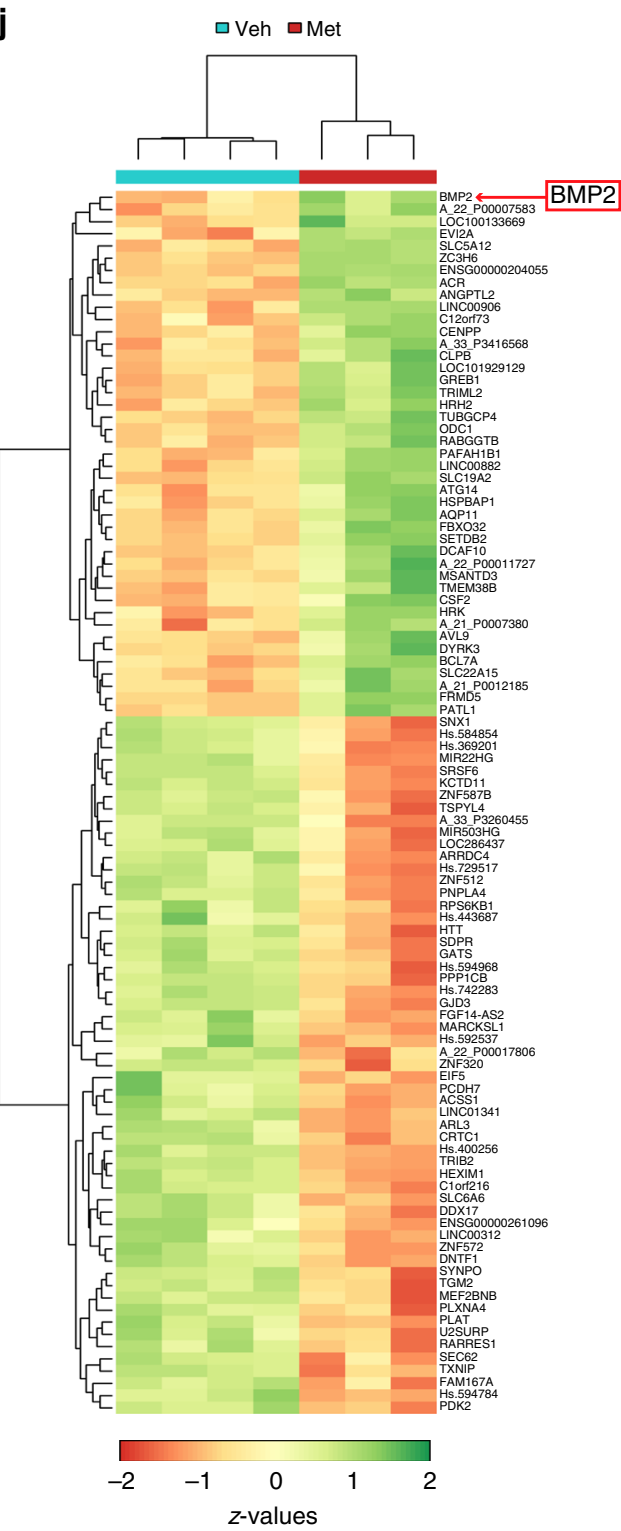

Fig. 1 Metformin induces lipogenic marker expression in human IPF lung fibroblasts. a Schematic representation of the experimental setup. b-d aPCR analysis for the lipogenic marker genes PLIN2 and PPARg, as well as the myofibroblast marker COL1A1 in human IPF lung fibroblasts treated with metformin or vehicle. e, $\mathbf{f}$ Staining of lipid droplets in fibroblasts using LipidTOX (red). Nuclei were counterstained with DAPI (blue). g-h Gating strategy for detecting LipidTOX ${ }^{+}$cells by flow cytometry. $\mathbf{i}$ Quantification of LipidTOX ${ }^{+}$cell abundance in response to metformin treatment. $\mathbf{j}$ Heatmap representation of the top 100 differentially expressed genes in fibroblasts following metformin treatment. $\mathbf{k}$ qPCR analysis for BMP2 in metformin- and vehicle-treated cells. Scale bars: e, $\mathbf{f} 25 \mu \mathrm{m}$. Each data point within a given group corresponds to one patient and error bars indicate s.e.m. b-d Vehicle-treated group: $n=12$, Metformin-treated group: $n=11$. i $n=3$ per group. $\mathbf{k} n=5$ per group. Student's $t$-test was used in (b-d) and Mann-Whitney test was used in (i, k). ${ }^{\star} P<0.05,{ }^{\star \star} P<0.01,{ }^{\star \star \star} P<0.001,{ }^{\star \star \star \star \star} P<0.0001$ 
consistent among all samples (i.e., induction of lipogenic marker expression and suppression of COL1A1 expression). As a final readout for lipogenic differentiation, the neutral lipid stain LipidTOX was used and the increase in lipid-droplet accumulation in fibroblasts was demonstrated by fluorescence microscopy (Fig. 1e, f). Flow cytometry-based quantification of LipidTOX ${ }^{+}$ cells displayed a significant 2.1 -fold increase in metformin-treated cells compared with vehicle-treated cells (Fig. 1g-i).

In order to explore global patterns of gene expression regulation induced by metformin and to generate further hypotheses, gene expression microarrays were carried out for four vehicle-treated and three metformin-treated human IPF lung fibroblast cultures. A heatmap depicting the top 100 differentially regulated genes is shown in Fig. 1j. KEGG pathway analysis revealed that metabolic pathways were the top modulated candidate in response to metformin treatment (Supplementary Fig. 3). The top 30 modulated signaling pathways included steroid biosynthesis, RNA degradation, vitamin B6 metabolism, glycosaminoglycan biosynthesis-heparan sulfate/heparin (which has previously been implicated in IPF $)^{22,23}$, PPAR signaling pathway, Hippo signaling pathway, terpenoid backbone biosynthesis, fatty acid metabolism, retinol metabolism, TNF signaling pathway, glycolysis/gluconeogenesis, and TGF $\beta$ signaling pathway (Supplementary Fig. 3). Other enriched pathways included biosynthesis of unsaturated fatty acids, glycerolipid metabolism, and fatty acid elongation (Supplementary Fig. 3). These findings support the notion that metformin induces lipogenic differentiation in human lung fibroblasts. We also inspected selected markers of lipogenic differentiation to validate the microarray data. In agreement with the qPCR data (Fig. 1b-d), we observed upregulation of PLIN2 and PPARg coupled to downregulation of the myofibroblast marker COL1A1 (Accession number GSE131133). Intriguingly, the top upregulated gene in metformin-treated fibroblasts was BMP2 (red arrow in Fig. 1j). This finding was validated by QPCR and the result indeed showed significant upregulation of BMP2 in metformin-treated cells compared with vehicle-treated controls (Fig. 1k).

Metformin inhibits TGF $\beta 1$-mediated fibrogenesis in vitro. The TGF $\beta 1$ signaling pathway is widely regarded as the master regulator of fibrogenesis in vitro and in vivo ${ }^{24}$. In order to investigate whether metformin inhibits the profibrotic effect of TGF $\beta 1$ in vitro, primary lung fibroblasts isolated from 10 IPF patients were starved for $24 \mathrm{~h}$ and then treated with $2 \mathrm{ng} / \mathrm{mL}$ rhTGF $\beta 1$ (or vehicle) for $72 \mathrm{~h}$ and then treated with $5 \mathrm{mM}$ metformin (or vehicle) for $72 \mathrm{~h}$ (Fig. 2a). This experimental setup allows examining the ability of metformin to change the phenotype of myofibroblasts that arise from TGF $\beta 1$ treatment. The effect of TGF $\beta 1$ treatment was validated after $72 \mathrm{~h}$ by $\mathrm{qPCR}$ and the results showed significant downregulation of the lipogenic markers PLIN2 (Fig. 2b) and PPARg (Fig. 2c) in parallel to significant upregulation of COL1A1 (Fig. $2 \mathrm{~d}$ ) as we previously described ${ }^{8}$. We also expected that activation of TGF $\beta 1$ signaling would result in disappearance of lipid droplets at the expense of ACTA2 and COL1A1 upregulation. To test this hypothesis, cells were stained with LipidTOX and anti-ACTA2 antibodies. Fluorescence microscopy showed increased abundance of ACTA2 filaments (Fig. 2h) and absence of lipid droplets (Fig. 2f) in TGF $\beta 1$-treated cells compared with vehicle-treated cells (Fig. 2e, g) after $72 \mathrm{~h}$. Time-lapse microscopy of vehicle- (Supplementary Movie 1), rhTGF 1 1- (Supplementary Movie 2) or metformin-treated cells (Supplementary Movie 3) in the presence of LipidTOX was also carried out for $68 \mathrm{~h}$. Metformin led to an increase in lipid-droplet accumulation after $48 \mathrm{~h}$ while vehicle or $\operatorname{rhTGF} \beta 1$ treatment led to cell elongation and disappearance of lipid droplets.
Next, the response to metformin treatment was analyzed. At the end of day 6 (144 h), qPCR showed significant upregulation of PLIN2 (4.6 folds, Fig. 2i) and PPARg (1.3 folds, Fig. 2j), and a significant 7.1-fold downregulation of COL1A1 expression (Fig. 2k) in the [TGF $\beta 1$ plus metformin] group compared with the [TGF $\beta 1$ plus vehicle] group. Interestingly, lipid-droplet accumulation was regained in fibroblasts treated with [TGF $\beta 1$ plus metformin] compared with the [TGF $\beta 1$ plus vehicle] group (Fig. 2l, m). These results suggest that metformin affects the phenotype of myofibroblasts arising from TGF $\beta 1$ treatment by downregulating myogenic markers and inducing lipogenic differentiation.

Metformin improves lung structure in an ex vivo system. One drawback of using cell-culture systems is that cells growing in vitro are deprived of their microenvironmental cues and their behavior might not resemble that of an in vivo context. We therefore set out to test whether metformin exerts similar beneficial effects in a more complex system that better mimics the in vivo setting. Therefore, we used precision-cut lung slices (PCLS), an ex vivo culture system that has been previously described $^{25}$. This technique allows maintenance of viable, metabolically active lung tissue with preserved structure for 5 days ${ }^{25}$. Two hundred micrometer-thick PCLS were prepared from fibrotic regions of fresh IPF lung tissues and were cultured in DMEM supplemented with $10 \%$ BCS in the presence or absence of $5 \mathrm{mM}$ metformin for 5 days (Fig. 3a). Bright-field imaging showed that metformin-treated PCLS displayed a more relaxed structure with open airspaces (Fig. 3e) compared with their vehicle-treated counterparts (Fig. 3d). PCLS were then embedded in paraffin, sectioned into 5 - $\mu \mathrm{m}$-thick slices, and processed for histological analysis. Hematoxylin and eosin staining (Fig. 3h, i), Masson's trichrome staining (Fig. 3j, k) and COL1A1 immunostaining (Fig. 3l, m) showed improved lung structure and decreased collagen deposition in metformin-treated samples compared with controls. Another set of PCLS was not sectioned but was subjected to whole-mount staining using anti-COL1A1 antibodies and LipidTOX followed by confocal microscopy. Three-dimensional (3D) reconstruction using z-stacks acquired from these samples revealed decreased COL1A1 expression and increased lipid-droplet accumulation (Fig. 3n, o). Flow cytometry-based quantification confirmed the histological observations and showed a significant increase in the abundance of lipid-droplet-containing cells (following depletion of CD45 ${ }^{+}$ bone marrow-derived cells, $\mathrm{CD}_{3} 1^{+}$endothelial cells and $\mathrm{EpCAM}^{+}$epithelial cells) in metformin-treated PCLS $(12 \pm 0.6 \%)$ compared with controls $(7.2 \pm 1.1 \%)$ (Fig. $3 p$, q). Finally, total collagen assay showed a significant reduction in collagen content from $26.9 \pm 0.9$ to $20.8 \pm 0.9 \mu \mathrm{g} / \mu \mathrm{L}$ in response to metformin treatment (Fig. 3r).

Metformin accelerates resolution of lung fibrosis in mice. We next sought to test whether the ability of metformin to alter the myofibroblast fate also applies to the in vivo context of lung fibrosis. In order to address this question, we employed a lineagetracing approach in the context of bleomycin-induced pulmonary fibrosis (Fig. 4). This model of injury is reversible and animals start to spontaneously recover from lung fibrosis after day 14. Moreover, we previously reported that the Acta2-Cre-ERT2; tdTomatoflox lineage-tracing tool allows genetic labeling of ACTA2 ${ }^{+}$myofibroblasts as they accumulate during the buildup of lung fibrosis when tamoxifen is introduced between days 5 and 14 after bleomycin injury ${ }^{8}$. Therefore, double transgenic animals were treated with bleomycin, fed tamoxifen-containing pellets between days 5 and 14, treated with metformin (or vehicle) 


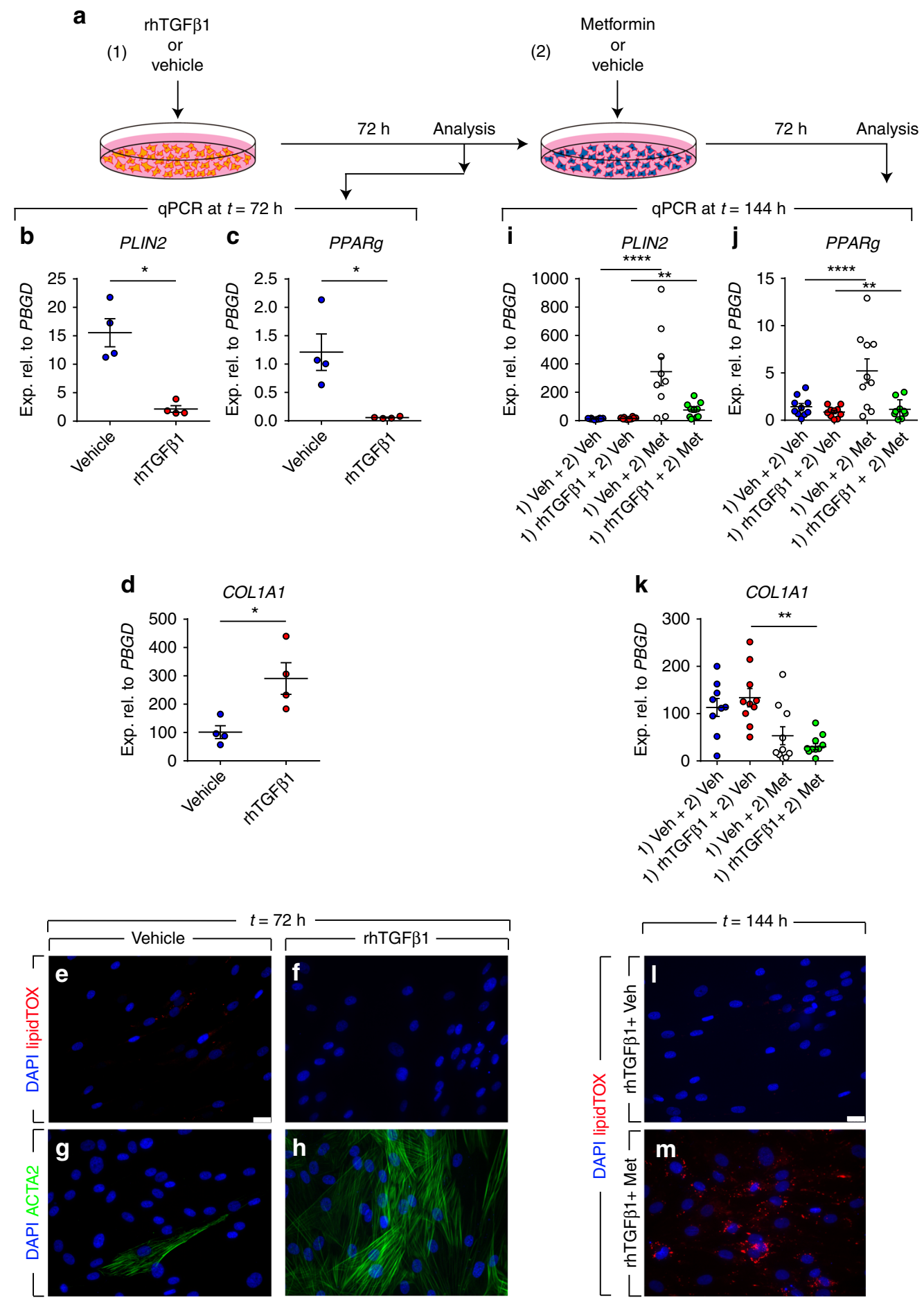

Fig. 2 Metformin attenuates TGFB1-mediated fibrogenesis in vitro. a Schematic representation of the experimental setup. b-d qPCR analysis for PLIN2, PPARg, and COLTA1 in human lung fibroblasts treated with TGF $\beta 1$ or vehicle for $72 \mathbf{h}$. e-h Staining of TGF $\beta 1$ - and vehicle-treated cells with LipidTOX (red), anti-ACTA2 antibodies (green), and DAPI (blue). i-k qPCR analysis for PLIN2, PPARg, and COL1A1 in human lung fibroblasts treated with TGF $\beta 1$ or vehicle for $72 \mathrm{~h}$, followed by treatment with metformin or vehicle for $72 \mathrm{~h}$. I, $\mathbf{m}$ Staining of TGF $\beta 1$ - and vehicle-treated cells with LipidTOX (red) and DAPI (blue) at the end of treatment $(t=144 \mathrm{~h})$. Scale bars: $(e-h)$ and $(\mathrm{l}, \mathrm{m}) 25 \mu \mathrm{m}$. Each data point within a given group corresponds to one patient and error bars indicate s.e.m. $\mathbf{b}-\mathbf{d} n=4$ per group. $\mathbf{i}-\mathbf{k} n=9-10$ per group. Mann-Whitney test was used in $(\mathbf{b}-\mathbf{d})$, one-way ANOVA was used in (i, $\mathbf{j})$ and Kruskal-Wallis test was used in $(\mathbf{k}) .{ }^{\star} P<0.05,{ }^{\star} * P<0.01,{ }^{\star \star \star \star} P<0.0001$. Met: metformin, Veh: vehicle

starting at day 14 after bleomycin instillation, and were sacrificed at day 28 (Fig. 4a, b). This experimental setup allows labeling myofibroblasts that form during fibrosis formation and tracing their fate during resolution in the presence or absence of metformin. Hematoxylin and eosin as well as Masson's trichrome staining of lungs collected at day 28 showed enhanced recovery from fibrosis upon metformin treatment (Fig. 4c-f). Correspondingly, quantification of lung fibrosis showed a significant decrease in the extent of fibrosis from $12 \pm 1.5 \%$ in the vehicletreated group to $8 \pm 0.6 \%$ in the metformin-treated group 


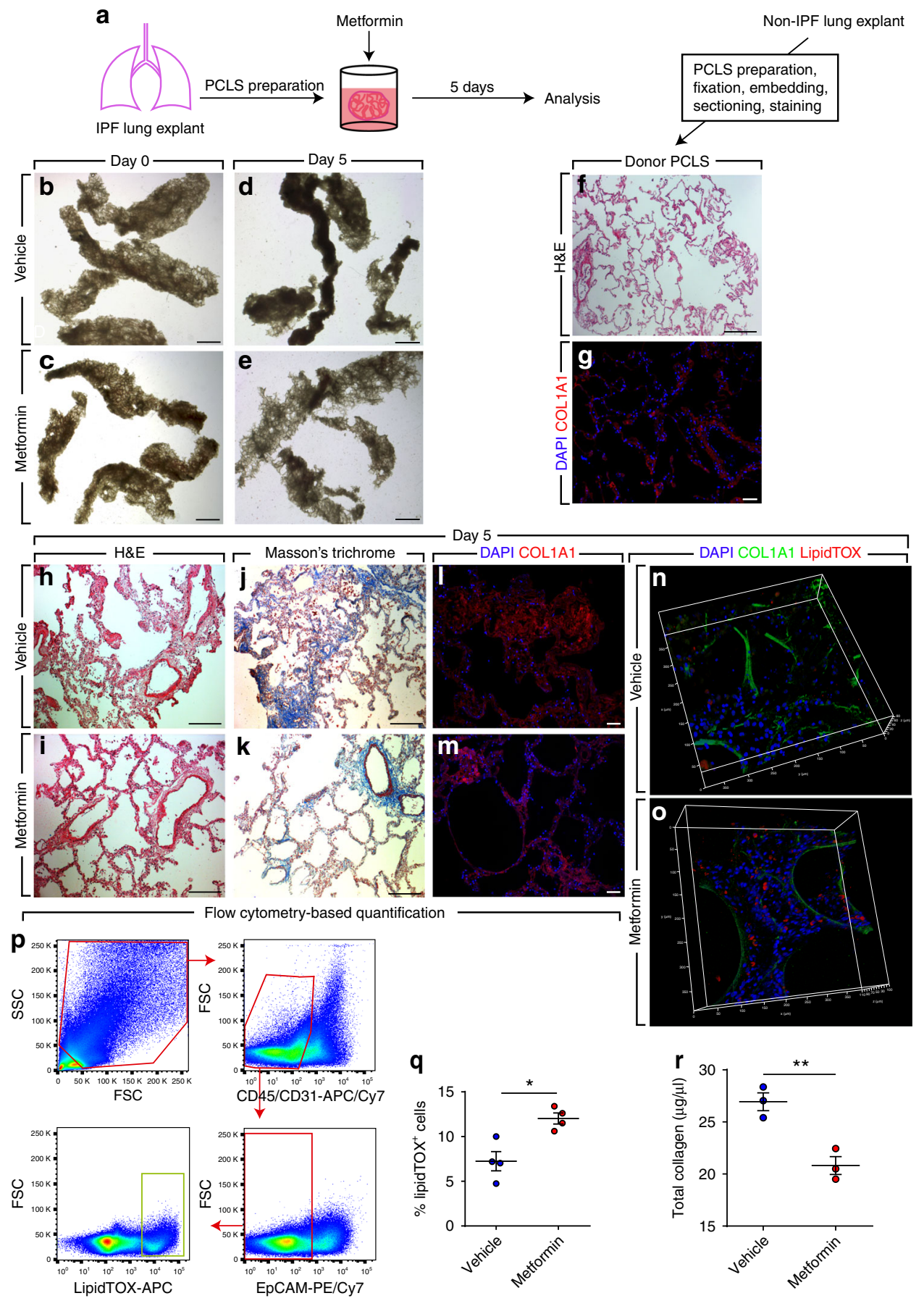

Fig. 3 Metformin improves IPF lung structure ex vivo. a Schematic representation of the experimental setup. b-e Bright-field imaging of PCLS treated with metformin or vehicle for 5 days. $\mathbf{f}, \mathbf{g}$ Hematoxylin and eosin staining and COL1A1 immunostaining of PCLS prepared from a non-IPF donor lung. $\mathbf{h}-\mathbf{m}$ Hematoxylin and eosin staining, Masson's trichrome staining and COL1A1 immunostaining of PCLS prepared from an IPF lung and treated with metformin or vehicle for 5 days. $\mathbf{n}$, o 3D reconstruction of z-stacks of metformin- and vehicle-treated PCLS stained for COL1A1 (green) and lipid droplets (red). p Gating strategy for flow cytometry-based quantification of LipidTOX+ cells that are negative for hematopoietic (CD45), endothelial (CD31), and epithelial (EpCAM) cell markers. q Quantification of flow cytometry measurements on metformin- and vehicle-treated cells. $\mathbf{r}$ Total collagen assay for metformin- and vehicle-treated cells. Scale bars: b-e $2 \mathrm{~mm}, \mathbf{f} 500 \mu \mathrm{m}, \mathbf{g}, \mathbf{l}, \mathbf{m} 50 \mu \mathrm{m}$, and $\mathbf{h}-\mathbf{k} 200 \mu \mathrm{m}$. Each data point within a given group corresponds to one patient and error bars indicate s.e.m. $\mathbf{q} n=4$ per group. $\mathbf{r} n=3$ per group. Mann-Whitney test was used in $(\mathbf{q}, \mathbf{r})$. ${ }^{\star} P<0.05,{ }^{\star} * 0<0.01$

(Fig. 4g). Immunofluorescence staining allowed visualization of lineage-traced myofibroblast-derived cells $\left(\right.$ tdTomato $^{+}$) and also confirmed the decrease in COL1A1 deposition upon metformin treatment (Fig. 4h, i). Interestingly, LipidTOX staining carried out on frozen sections revealed the presence of lipid droplets in tdTomato $^{+}$cells at day 28 (Fig. $4 \mathrm{j}, \mathrm{k}$ ). In order to quantify the impact of metformin on altering the myofibroblast fate, flow cytometry was carried out. The results showed similar proportions of tdTomato ${ }^{+}$cells in both groups (Fig. 4q), while the proportion of LipidTOX ${ }^{+}$mesenchymal cells increased from $13.5 \pm 2.5 \%$ to $18.8 \pm 1.8 \%$ upon metformin treatment (Fig. $4 \mathrm{~s}$ ). More importantly, the proportion of myofibroblast descendants $\left(\right.$ tdTomato $\left.^{+}\right)$that also contained lipid droplets increased from $6.8 \pm 0.1 \%$ in the control group to $12.2 \pm 0.5 \%$ in the 


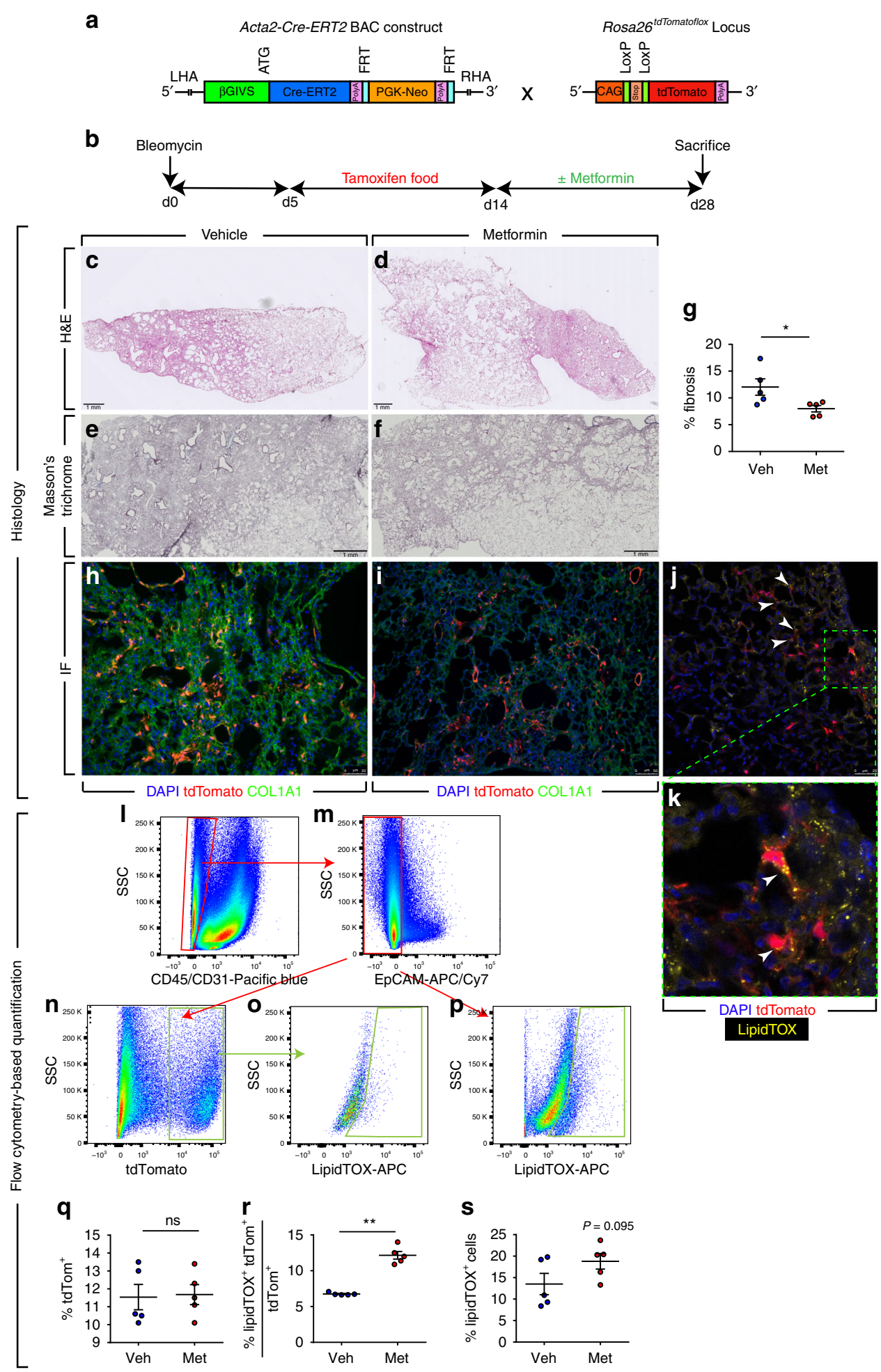

Fig. 4 Metformin accelerates fibrosis resolution in the bleomycin model in mice. a Schematic representation of the Acta2-Cre-ERT2 and tdTomatoflox constructs. b Schematic representation of the timeline of the experiment. Bleomycin was administered intratracheally at day 0 . Between days 5 and 14 , mice were fed tamoxifen-containing pellets and starting at day 14 , metformin $(1.5 \mathrm{mg} / \mathrm{mL})$ or vehicle was administered through drinking water. Mice were sacrificed at day 28. c-f Hematoxylin and eosin and Masson's trichrome staining of metformin- and vehicle-treated lungs. $\mathbf{g}$ Quantification of fibrosis in metformin- and vehicle-treated lungs. h, i Immunofluorescence for COL1A1 (green). Endogenous tdTomato signal (red) and DAPI (blue) are also shown. j LipidTOX staining (green) and tdTomato ${ }^{+}$cells (red) are shown. The box in ( $\mathbf{j}$ ) is magnified in (k). Arrowheads indicate LipidTOX ${ }^{+} \mathrm{tdTomato}^{+}$cells. I-s Gating strategy (to detect CD45- CD31- EpCAM- tdTomato ${ }^{+}$and/or LipidTOX ${ }^{+}$cells) and quantification of various cell populations based on tdTomato and LipidTOX detection. Scale bars: c-f $1 \mathrm{~mm}, \mathbf{h}, \mathbf{i} 50 \mu \mathrm{m}$, and $\mathbf{j} 25 \mu \mathrm{m}$. Each data point within a given group corresponds to one animal and error bars indicate s.e.m. $n=5$ per group. Mann-Whitney test was used in $(\mathbf{g}, \mathbf{q}-\mathbf{s}) .{ }^{\star} P<0.05,{ }^{\star \star} P<0.01$. IF: immunofluorescence, ns: not significant 

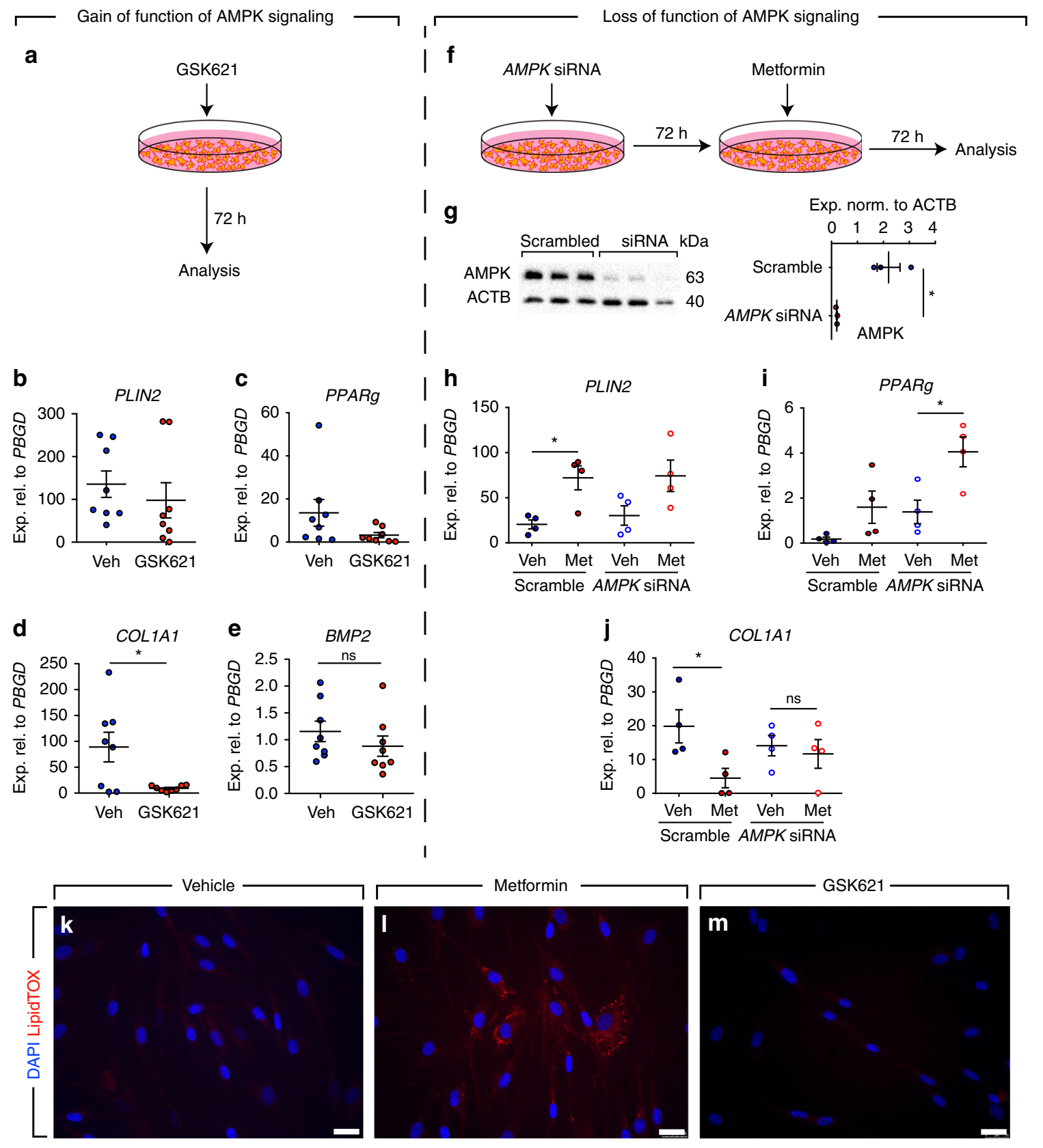

Fig. 5 Mode of action of metformin is partially independent of AMPK signaling. a Schematic representation of the gain-of-function experimental setup for AMPK signaling. b-e qPCR analysis of PLIN2, PPARg, COL1A1, and BMP2 in human IPF lung fibroblasts treated with the AMPK agonist GSK621 or vehicle. f Schematic representation of the loss-of-function experimental setup for AMPK signaling. The decrease of AMPK protein levels at the time of analysis is shown in (g). $\mathbf{h}-\mathbf{j}$ qPCR analysis of PLIN2, PPARg, and COL1A1 in IPF fibroblasts treated with AMPK siRNA or scramble siRNA. k-m Staining of GSK621- and vehicle-treated cells with LipidTOX (red) and DAPI (blue). Metformin-treated cells were used as a positive control for lipid-droplet accumulation (I). Scale bars: $\mathbf{k}-\mathbf{m} 25 \mu \mathrm{m}$. Each data point corresponds to one patient and error bars indicate s.e.m. b-e Vehicle-treated group: $n=8$, GSK621-treated group: $n=8$. $\mathbf{g} n=3$ per group. $\mathbf{h}-\mathbf{j} n=4$ per group. Mann-Whitney test was used in $(\mathbf{b}-\mathbf{e}, \mathbf{g})$ and Kruskal-Wallis test was used in $(\mathbf{h}-\mathbf{j})$. ${ }^{\star} P<0.05$. ns: not significant

metformin-treated group (Fig. 4r). Collectively, these data clearly demonstrate that metformin accelerates fibrosis resolution and this is accompanied by the induction of myogenic-to-lipogenic conversion in lung fibroblasts in vivo.

AMPK signaling does not impact lipogenic differentiation. Activation of the AMPK signaling cascade is one of the major molecular mechanisms that have been proposed for metformin. In order to investigate whether metformin-mediated lipogenic differentiation is AMPK-dependent, gain- and loss-of-function approaches were carried out. In a first set of experiments, primary human lung fibroblasts isolated from 8 IPF patients were cultured and treated with GSK621 (a selective activator of AMPK signaling pathway) for $72 \mathrm{~h}$ (Fig. 5a). The results showed a trend for mild downregulation of the lipogenic markers PLIN2 (Fig. 5b) and PPARg (Fig. 5c) in parallel to a robust, significant downregulation of COL1A1 (Fig. 5d). GSK621 treatment did not affect BMP2 expression in these cells (Fig. 5e). Accordingly, GSK621 treatment failed to promote lipid-droplet accumulation in these cells (Fig. $5 \mathrm{k}-\mathrm{m}$ ).

In another set of experiments, primary human lung fibroblasts isolated from five IPF patients were transfected with 
siRNAs targeting $A M P K$. After $72 \mathrm{~h}$, cells were treated with metformin and were analyzed after $72 \mathrm{~h}$ (Fig. 5f). Western blotting revealed a $90 \%$ knockdown of $A M P K$ in these cells compared with scramble-transfected cells at the time of analysis (Fig. 5g). Interestingly, metformin treatment led to upregulation of PLIN2 and PPARg regardless of AMPK knockdown (Fig. 5h, i). Metformin-mediated COL1A1 downregulation, however, was attenuated upon $A M P K$ knockdown (Fig. 5j). Altogether, these data demonstrate that the mechanism of action of metformin in promoting lipogenic differentiation in human lung fibroblasts is largely independent of AMPK signaling while inhibition of COL1A1 production is mainly AMPK-dependent.

BMP2 and PPAR $\gamma$ signaling mediate lipogenic differentiation. The next step was to better define the molecular mechanism by which metformin induces lipogenic differentiation in human lung fibroblasts. Our gene expression microarrays showed that $B M P 2$ was the top upregulated gene in fibroblasts upon metformin treatment (Fig. 1j, k). BMP2 is known to inhibit smooth muscle cell growth in a mechanism involving PPAR $\gamma$ activation $^{26}$. Calvier et al. also reported that BMP2 inhibits TGF $\beta 1$ signaling via PPAR $\gamma$ in vascular smooth muscle cells in the lung 27 . Therefore, we treated 11 human IPF lung fibroblasts with rhBMP2 and gene expression was analyzed after $72 \mathrm{~h}$ (Fig. 6a). Intriguingly, rhBMP2 treatment resulted in significant upregulation of PLIN2 (1.9 folds, Fig. 6b) and PPARg (2.5 folds, Fig. 6c), while COL1A1 expression levels remained unchanged (Fig. 6d). LipidTOX staining confirmed the increase in lipiddroplet accumulation in these cells (Fig. 6e, f). We then performed siRNA-mediated knockdown of PPARg in human IPF lung fibroblasts (Fig. $6 \mathrm{~g}$ ) and tested the effect of rhBMP2 treatment on lipogenic differentiation. The results showed that PPARg knockdown abolished rhBMP2-induced PLIN2 upregulation in these cells (Fig. 6h). As expected, COL1A1 levels were not modulated by this intervention (Fig. 6j). Thus, BMP2 is a positive regulator of lipogenic differentiation in human IPF lung fibroblasts and its mechanism of action involves PPAR $\gamma$ signaling. The activation of PPAR $\gamma$ signaling pathway can also be induced post-translationally via PPAR $\gamma$ phosphorylation at serine residue $112\left(\mathrm{Ser}^{112}\right)^{28-30}$. Therefore, we set out to determine whether BMP2 induces lipogenic differentiation in human lung fibroblasts by inducing PPAR $\gamma$ phosphorylation. Cells were treated with rhBMP2 (or vehicle) and protein lysates were collected after $72 \mathrm{~h}$. PPAR $\gamma$ phosphorylation was induced 7.6 folds in rhBMP2-treated fibroblasts while PPAR $\gamma$ protein levels remained unchanged (Fig. 6k).

In another set of experiments, treatment of cells with TGF $\beta 1$ led to a 24.4-fold reduction in p-PPAR $\gamma$ levels (Fig. 7a, lanes 4-6 and Fig. 7b) while treatment with metformin led to a 3.3-fold increase in p-PPAR $\gamma$ levels (Fig. 7a, lanes 7-9 and Fig. 7b) compared with vehicle-treated cells (Fig. 7a, lanes 1-3 and Fig. 7b). In order to investigate whether metformin reverts the profibrotic effects of TGF $\beta 1$ in fibroblasts by modulating the phosphorylation status of PPAR $\gamma$, cells were first starved for $24 \mathrm{~h}$ and then treated with rhTGF $\beta 1$ for $72 \mathrm{~h}$, followed by vehicle, rhTGF $\beta 1$ or metformin treatment for another $72 \mathrm{~h}$. The results showed that in comparison to transient (Fig. 7a, lanes 10-12) or continuous treatment with rhTGF $\beta 1$ (Fig. 7a, lanes 13-15), treatment with metformin after $\operatorname{rhTGF} \beta 1$ treatment rescued $\mathrm{p}$ PPAR $\gamma$ levels (Fig. 7a, lanes $16-18$ and Fig. 7c). Of note, qPCR analysis revealed that activation of AMPK signaling via GSK621 treatment did not induce $B M P 2$ expression in IPF fibroblasts (Fig. 5e), indicating that the metformin-BMP2-p-PPAR $\gamma$ axis is independent of AMPK signaling.
In order to demonstrate that metformin-induced lipogenic differentiation is mediated by BMP2 signaling, cells were treated with metformin in the presence or absence of the BMP inhibitor noggin. The levels of $\mathrm{p}$-SMAD1/5/8 were used as readout for BMP2 signaling. Treatment with metformin led to significant phosphorylation of SMAD1/5/8 (Fig. 7d, lanes 7-10 and Fig. 7e), indicating that metformin treatment indeed activates BMP2 signaling. As expected, treatment with vehicle (Fig. 7d, lanes 1-3 and Fig. 7e), noggin (Fig. 7d, lanes 4-6 and Fig. 7e) or metformin + noggin (Fig. 7d, lanes 11-14 and Fig. 7e) did not yield significant SMAD1/5/8 phosphorylation. In line with these data, qPCR analysis revealed that noggin treatment abolished metformin-mediated upregulation of PLIN2 (Fig. 7f) and PPARg (Fig. 7g) without affecting metformin-mediated downregulation of COL1A1 (Fig. 7h). In another set of experiments, pre-treatment of the cells with $B M P 2$ siRNA abolished metformin-induced lipogenic differentiation without affecting COL1A1 downregulation (Supplementary Fig. 4). Altogether, these results indicate that metformin-induced lipogenic differentiation, but not collagen reduction, is mediated by BMP2 signaling.

In an attempt to explore modulations in serine/threonine protein kinase activities upon metformin treatment, human IPF lung fibroblasts were subjected to a protein kinase activity assay (PamStation). The results showed significantly reduced kinase activities for CDK1, CDK3, MAPK11/14, and ERK1/2 (Supplementary Fig. 5A). CDK7 and CDK9 did not show a conclusive activity profile. Given the relevance of ERK1/2 in lung fibrosis ${ }^{31}$ and since PPAR $\gamma$ can serve as a substrate for MAPK ${ }^{28,32}$, we validated the effect of metformin in inhibiting the activity of ERK1/2 by western blotting and the results showed robust inhibition of $p$-ERK1/2 levels in IPF lung fibroblasts in response to metformin treatment (Supplementary Fig. 5B). Treatment of human IPF lung fibroblasts with Selumetinib (a potent and selective MEK inhibitor) did not yield significant changes in the expression levels of lipogenic or myogenic marker genes (Supplementary Fig. 5C-F). Therefore, the ERK pathway does not seem to impact metforminmediated myo- to lipofibroblast transdifferentiation. The identity of the protein kinase(s) responsible for PPAR $\gamma$ phosphorylation at Ser ${ }^{112}$ and induction of lipogenic differentiation in response to metformin or BMP2 treatment warrants further investigations.

Pirfenidone and nintedanib do not induce lipogenesis. Currently, there are two FDA-approved drugs for treating IPF; pirfenidone, which is believed to act as a TGF $\beta 1$ inhibitor, and nintedanib, which is a multi-receptor tyrosine kinase inhibitor. Therefore, we set out to determine whether these two drugs alter lung fibroblast fate in a similar fashion to metformin. First, primary human lung fibroblasts were treated with either nintedanib $(1 \mu \mathrm{M})$ or pirfenidone $(0.3 \mathrm{mg} / \mathrm{ml})$ for $72 \mathrm{~h}$ (Supplementary Fig. 6A). Quantitative real-time PCR analysis showed that neither nintedanib nor pirfenidone enhanced the expression of the lipogenic markers PLIN2 and PPARg (Supplementary Fig. 6B, C, E, F). Nintedanib and pirfenidone did, however, lead to a 1.9- and 2.3fold downregulation of $C O L 1 A 1$, respectively (Supplementary Fig. 6D, G). In line with these data, LipidTOX staining did not reveal any change in lipid-droplet accumulation following nintedanib or pirfenidone treatment (Supplementary Fig. 6H-J). Finally, whole-mount staining followed by confocal imaging and $3 \mathrm{D}$ reconstruction of IPF-derived PCLS (cultured for 5 days in the presence or absence of nintedanib or pirfenidone) revealed a slight decrease in COL1A1 deposition without an evident change in the abundance of LipidTOX ${ }^{+}$cells (Supplementary Fig. 6K-O). 
a
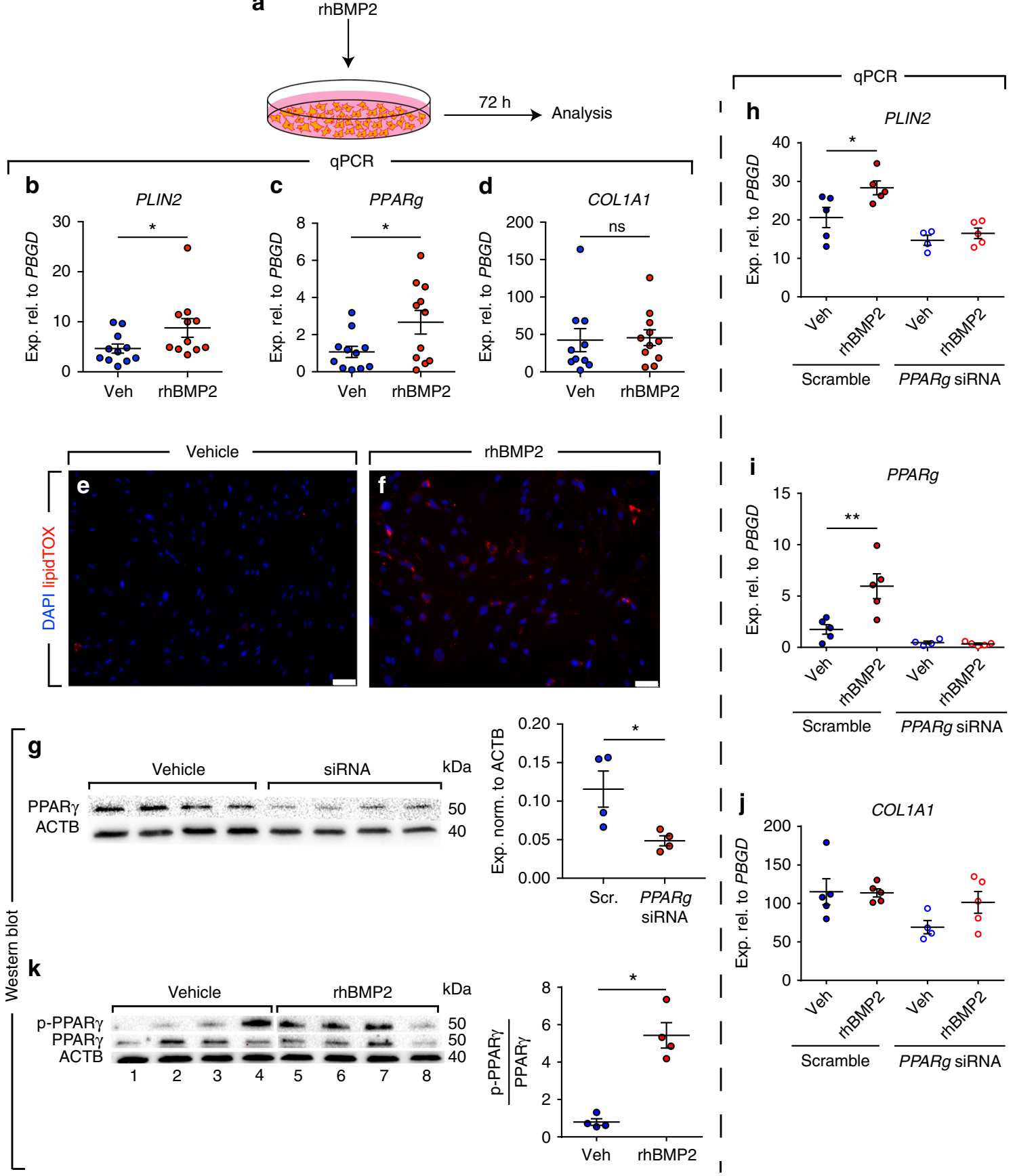

Fig. 6 rhBMP2 induces PPAR $\gamma$ phosphorylation and lipogenic differentiation in human IPF lung fibroblasts. a Schematic representation of the experimental setup. b-d qPCR analysis of PLIN2, PPARg and COL1A1 in IPF fibroblasts treated with rhBMP2 or vehicle. e, $\mathbf{f}$ Staining of rhBMP2- and vehicle-treated cells with LipidTOX (red) and DAPI (blue). $\mathbf{g}$ Western blot validating the knockdown of PPAR $\gamma$ protein levels $72 \mathrm{~h}$ after siRNA treatment. Quantification of the immunoblot is shown in the right panel. $\mathbf{h}-\mathbf{j}$ qPCR analysis of PLIN2, PPARg, and COL1A1 in IPF fibroblasts transfected with siRNA against PPARg (for $72 \mathrm{~h}$ ) and then treated with vehicle or rhBMP2 for $72 \mathrm{~h}$. $\mathbf{k}$ Western blot showing the induction of PPAR $\gamma$ phosphorylation in response to rhBMP2 treatment. Lanes 1-4 and lanes 5-8 were run in parallel on different gels under the same conditions. Quantification of the immunoblot is shown in the right panel. Scale bars: e-f $50 \mu \mathrm{m}$. Each data point corresponds to one patient and error bars indicate s.e.m. b-d $n=11$ per group except for COL1A1 vehicle-treated group ( $n=10$ ). $\mathbf{g}, \mathbf{k} n=4$ per group. $\mathbf{h}-\mathbf{j}$ Scramble/vehicle-, scramble/rhBMP2- and siRNA/rhBMP2-treated groups: $n=5$ per group, siRNA/vehicle-treated group: $n=4$. Mann-Whitney test was used in $(\mathbf{b}, \mathbf{d}, \mathbf{g}, \mathbf{k})$ and Student's $t$-test was used in $(\mathbf{c})$. Kruskal-Wallis test was used in $(\mathbf{h}-\mathbf{j}) .{ }^{\star} P<0.05,{ }^{\star \star} P<0.01$. ns: not significant

\section{Discussion}

Despite recent advances in our understanding of IPF pathology, there is still no curative treatment for IPF, and the currently available antifibrotic treatment modalities retard, but do not completely stop, the progression of the disease. On the other hand, there is emerging literature about the association between metabolic disorders and IPF incidence. We have recently demonstrated an interconversion between lipogenic and myogenic fibroblastic phenotypes in lung fibrosis, a process that is governed by TGF $\beta 1$ and PPAR $\gamma$ signaling pathways ${ }^{8}$. We and others had already shown that the PPAR $\gamma$ agonist rosiglitazone, which is an antidiabetic agent, counteracts TGF $\beta 1$-mediated 


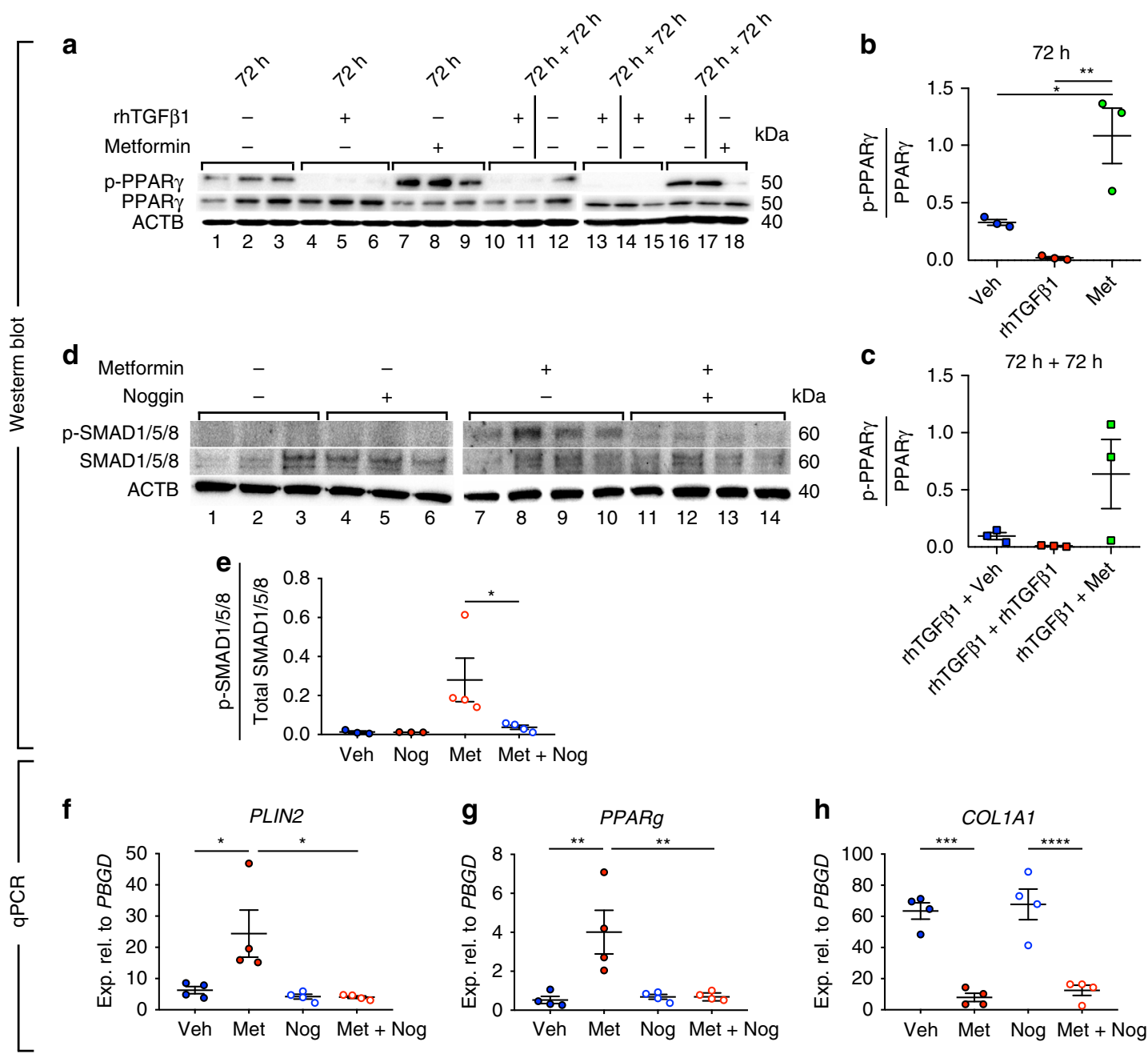

Fig. 7 Metformin-mediated lipogenic differentiation in human IPF lung fibroblasts is mediated by BMP2 signaling. a-c Western blot showing the opposing effects of metformin and rhTGF $\beta 1$ on PPAR $\gamma$ phosphorylation and the ability of metformin to partially restore PPAR $\gamma$ phosphorylation in rhTGF $\beta 1$-treated IPF fibroblasts. Lanes 1-12 and lanes 13-18 were run in parallel on different gels under the same conditions. $\mathbf{d}$ Western blot showing the phosphorylation status of SMAD1/5/8 in the presence of metformin and/or the BMP signaling inhibitor noggin. Quantification of the immunoblot is shown in (e). $\mathbf{f}-\mathbf{h}$ qPCR analysis of PLIN2, PPARg, and COL1A1 in IPF fibroblasts treated with vehicle, metformin, noggin and noggin + metformin. Each data point corresponds to one patient and error bars indicate s.e.m. a-c $n=3$ per group. d Vehicle- and noggin-treated groups: $n=3$ per group, metformin- and metformin/noggintreated groups: $n=4$. $\mathbf{f}-\mathbf{h} n=4$ per group. Kruskal-Wallis test was used in $(\mathbf{b}, \mathbf{c}, \mathbf{e}-\mathbf{h}) .{ }^{\star} P<0.05,{ }^{\star \star} P<0.01,{ }^{\star \star \star} P<0.001,{ }^{\star \star \star \star} P<0.0001$

fibrogenesis in vitro and in vivo ${ }^{8-10}$. In this study, we demonstrate that the first-line antidiabetic drug, metformin, inhibits collagen production in primary human lung fibroblasts and in ex vivo cultured human IPF PCLS and strongly enhances myo- to lipofibroblast transdifferentiation linked with phenotypic recovery from fibrosis. Although the PCLS culture system does not fully recapitulate the in vivo situation, it adds cellular, molecular and matrix complexity compared with standard cell-culture techniques and-in our opinion-offers a valuable preclinical analytical tool. Accordingly, therapeutic application of metformin in bleomycin-injured mice resulted in accelerated resolution of fibrosis by altering the fate of myofibroblasts and inducing their lipogenic differentiation. In our in vivo experiments, metformin was introduced through drinking water and the effects on lung repair were evident based on fibrosis assessment and myofibroblast fate switching. No adverse, systemic side effects were observed. Although metformin is safe and well tolerated in humans, it confers the risk of hypoglycemia in case of long-term use, although this risk is still lower than that associated with other antidiabetic agents ${ }^{33}$. Notably, detailed pathway analysis showed that the reduction of collagen synthesis was largely AMPKdependent, whereas the transdifferentiation of myo- to lipofibroblasts occurred in a BMP2-PPAR $\gamma$-dependent fashion and was largely AMPK-independent.

PPAR $\gamma$ is the master regulator of adipogenesis and it is expressed in various cell types in the human body. Its functions include - in addition to differentiation and maintenance of adipocytes ${ }^{34}$-regulation of inflammatory responses in macrophages $^{35}$, regulation of osteogenesis ${ }^{36}$, and cell metabolism ${ }^{37}$. Therefore, its role in homeostasis and disease is strictly contextdependent. PPAR $\gamma$ phosphorylation has been shown to act as an activation or inhibitory signal depending on the protein kinase involved and the cell type being studied. An earlier study has shown that phosphorylation of Ser ${ }^{112}$ in the N-terminal transactivation domain of PPAR $\gamma$ by MAPK acts as an activation signal in Chinese hamster ovary $(\mathrm{CHO})$ cells $^{28}$. Another study has shown that the same phosphorylation event acts as an inhibitory signal in white adipose tissue (WAT) ${ }^{32}$. Intriguingly, phosphorylation of the same serine residue in WAT by cyclin-dependent kinase 7 (CDK7) acts as an activation signal ${ }^{29}$, and so does 
phosphorylation by CDK9 in the embryonic mouse preadipocyte cell line $3 \mathrm{~T} 3-\mathrm{L}_{1}^{30}$. Therefore, it is clear that the outcome of phosphorylation in terms of PPAR $\gamma$ activation and consequently lipogenic differentiation is context-dependent. In this study, we found that metformin significantly upregulates $B M P 2$ and $P P A R g$ (at the mRNA level), increases the phosphorylation of PPAR $\gamma$ protein at $\mathrm{Ser}^{112}$, upregulates the downstream target PLIN2 $2^{38,39}$ and induces lipid-droplet accumulation in human IPF lung fibroblasts. Interestingly, metformin has been reported to inhibit adipogenesis in mesenchymal stem cells ${ }^{40}$ and 3T3-L1 preadipocytes ${ }^{41}$. Therefore, the biological and physiological outcome of metformin treatment is also context- and cell type-specific.

Recently, two independent groups have shown that metformin inhibits the profibrotic effect of TGF $\beta 1$ in lung fibroblasts via AMKP activation ${ }^{20,21}$. Our data are in line with these reports and they reveal an additional mechanism by which metformin acts on lung fibroblasts to attenuate fibrosis. Our gain-of-function experiments showed that while AMPK activation per se downregulated $C O L 1 A 1$, it was not enough to trigger the transdifferentiation of myofibroblasts into lipofibroblasts. Correspondingly, knockdown of $A M P K$ did not abolish metformin-mediated induction of lipogenic markers but attenuated the suppression of COL1A1 expression. These data suggest that alternative signaling mechanisms also contribute to the antifibrotic effects of metformin. Our data strongly suggest that BMP2 upregulation and PPAR $\gamma$ phosphorylation are centrally involved in these mechanisms. While rhBMP2 treatment was sufficient to phosphorylate PPAR $\gamma$ and induce lipogenic marker expression in primary human IPF lung fibroblasts, rhBMP2 treatment did not result in COL1A1 downregulation. In addition, inhibition of BMP2 signaling suppressed metformin-induced lipogenic differentiation without affecting the ability of metformin to inhibit collagen production, and knockdown of PPARg abolished the ability of BMP2 to induce lipogenic differentiation. Therefore, we propose a model in which metformin firstly activates AMPK signaling, which downregulates COL1A1, and secondly activates an alternative pathway involving $B M P 2$ upregulation and PPAR $\gamma$ phosphorylation, which induces lipogenic differentiation (Fig. 8).

Myofibroblast-to-lipofibroblast trans- or redifferentiation is a central hitherto largely underappreciated route for resolution of lung fibrosis. Side-by-side comparison of metformin with pirfenidone and nintedanib showed that the latter agents did not converge on this resolution route although the cells and tissues used for treatment with either compound were derived from the same patients. Metformin, like rosiglitazone and maybe other antidiabetic medications, may add a unique antifibrotic profile by inducing transdifferentiation of myo- to lipofibroblasts. Another likely possibility is that since IPF lung tissues are derived from end-stage patients that underwent lung transplantation, it might be that these samples had developed resistance to pirfenidone and nintedanib. Nevertheless, the robust response of these samples to metformin treatment in terms of COL1A1 downregulation and lipogenic differentiation highlights the therapeutic potential of metformin in IPF.

Metformin is known to manipulate various metabolic pathways $^{42-44}$. Metformin is known to disrupt mitochondrial complex I, thus inhibiting cellular respiration. Recently, Zhao et al. published an analysis of metabolic alterations in IPF lungs compared with donors ${ }^{14}$. They concluded that several types of long- and medium-chain fatty acids are enriched in IPF lungs ${ }^{14}$. Sphingolipid metabolism was found to be suppressed while arginine metabolism was found to be enhanced in the IPF lungs ${ }^{14}$. Our KEGG analysis on human lung fibroblasts suggests that metformin might be able to correct such metabolic dysregulations. Better understanding of the metabolic switch in lung fibroblasts in response to metformin and the mechanisms leading to accumulation of lipid droplets warrants further research. In addition, we cannot exclude that the beneficial effect of metformin in the context of lung fibrosis could also be attributed to direct effects on AT2, rather than solely mediated by effects on mesenchymal cells. In the future, it would be extremely important to establish a mesenchyme-free AT2 culture model (derived from IPF lungs) and test the effect of metformin, as well as other therapeutic candidates, in this context.

Post-hoc analysis was recently performed on IPF patient data derived from the placebo arms of three phase 3, double-blind, controlled trials of pirfenidone ${ }^{45}$. In that study, 71 metformin users did not present improvements in clinical outcomes compared with 553 non-metformin users ${ }^{45}$. As pointed out by Tzouvelekis and colleagues, these data cannot be generalized into the global IPF population due to many caveats ${ }^{46}$. Given its low cost and the fact that it is well tolerated in humans, it will be useful to test the curative effect of metformin, either alone or in combination with other antifibrotic agents, in non-diabetic IPF patients. A key aspect will be to identify biomarkers that predict drug responsiveness in the heterogeneous population of IPF patients. Given the high financial burden of developing novel drugs, drug repositioning might help accelerate the process of discovering a cure for IPF.

To sum up, our data demonstrate a clear antifibrotic role for metformin in the lung. Due to its ability to alter metabolic pathways, to inhibit TGF $\beta 1$ signaling, to suppress collagen formation and to promote transdifferentiation of myo- into lipofibroblasts, metformin should be considered as a therapeutic option for IPF patients.

\section{Methods}

Human-derived specimens. Fresh human lung explants and primary lung fibroblasts were obtained through the European IPF registry (eurIPFreg) at the Universities of Giessen and Marburg Lung Center, member of the German Center for Lung Research ${ }^{47}$. Written consent was obtained from each patient and the study was approved by the ethics committee of Justus-Liebig University Giessen.

Animal experiments. All animals were housed under specific pathogen-free (SPF) conditions with free access to food and water. Acta2-Cre-ERT2 (STOCK_Tg(Acta2cre/ERT2)12Pcn) mice ${ }^{48}$ were a kind gift from Dr. Pierre Chambon (University of Strasbourg, France). In this transgenic line, Cre-ERT2 coding sequence was inserted at the initiation codon of Acta2 in a mouse bacterial artificial chromosome (BAC) (MGI: 3831907). The Cre reporter line tdTomato flox (B6;129S6-Gt(ROSA) 26Sortm9(CAG-tdTomato)Hze/J was purchased from the Jackson laboratory (stock number 007909) ${ }^{49}$. At 11-12 weeks of age, Acta2-Cre-ERT2; tdTomato flox males were subjected to intratracheal instillation of saline or bleomycin $(0.8 \mathrm{U} / \mathrm{kg}$ body weight) (Sigma-Aldrich) using a micro-sprayer (Penn-Century, Inc.) at the Ludwig Boltzmann Institute in Graz, Austria. Five days after bleomycin instillation, mice were fed tamoxifen-containing chow $(400 \mathrm{mg} / \mathrm{kg}$ food, Envigo) for 9 days. Metformin $(1.5 \mathrm{mg} / \mathrm{mL})$ or vehicle (PBS) was supplied via drinking water at day 14 after bleomycin instillation. Lungs were harvested on day 28. All animal experiments were approved by the local authorities (Austrian Ministry of Education, Science and Culture; BMWFW-66.010/0043-WF/V/3b/2016) and performed in accordance with the EU directive 2010/63/EU.

Cell culture. Primary lung fibroblasts derived from 12 IPF patients were main tained in Dulbecco's modified Eagle's medium (DMEM) (Invitrogen) supplemented with $10 \%$ bovine calf serum (BCS, Gibco) at $37^{\circ} \mathrm{C}$ and $5 \% \mathrm{CO}_{2}$. Cells between passages 3 and 7 were used for the experiments. Briefly, $3 \times 10^{5}$ cells were seeded per well in 6-well plates (Greiner Bio-One). The next day, cells were starved ( $0 \%$ serum) for $24 \mathrm{~h}$ and then treated with different compounds. For imaging experiments, cells were cultured in 4 -well chamber slides (Sarstedt) at a density of 75,000 cells per well and were treated according to the same procedure described above. Cells were treated with metformin (Ratiopharm), pirfenidone (Cayman Chemical Company), nintedanib, selumetinib or GSK621 (all from Selleckchem), recombinant human TGF $\beta 1$ (rhTGF $\beta 1$ ), rhBMP2 or noggin (all from R\&D Systems). Table 1 summarizes treatment conditions. As controls, the same cells were treated with the corresponding solvents as recommended by the manufacturer (same volumes as treated groups).

Precision-cut lung slices. Fresh lung specimens were obtained from four IPF patients that underwent lung transplantation. Precision-cut lung slices were 


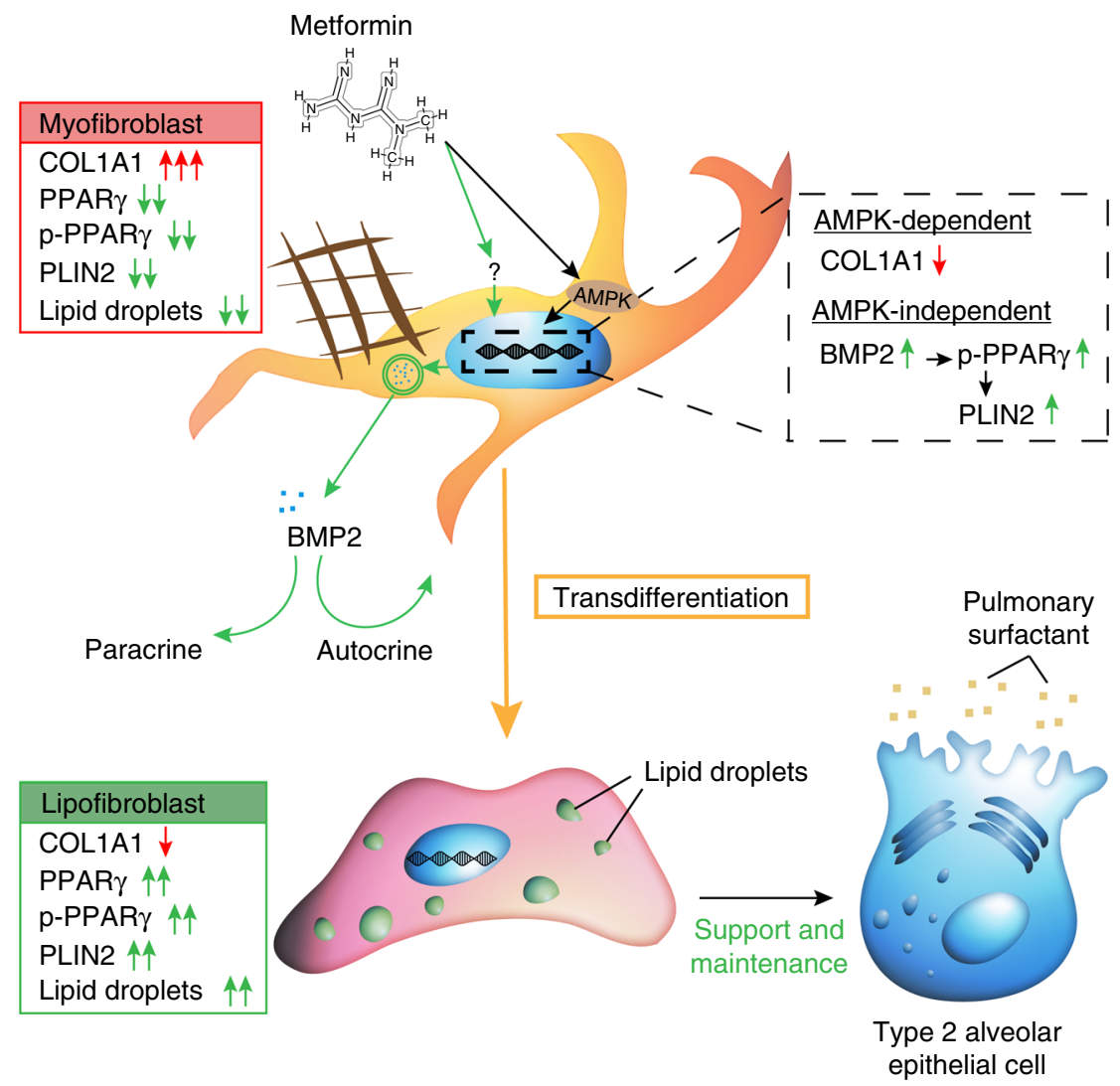

Fig. 8 Model for the antifibrotic mechanism of action of metformin in human lung fibrosis. Metformin activates AMPK signaling in myofibroblasts, leading to suppression of collagen production, and induces lipogenic differentiation via an AMPK-independent mechanism involving BMP2 release and PPAR $\gamma$ phosphorylation. Arising lipofibroblasts are known to support type 2 alveolar epithelial stem cells in the lung

\begin{tabular}{|c|c|c|}
\hline Compound & Final concentration & Solvent \\
\hline GSK621 & $10 \mu M^{a}$ & DMSO \\
\hline Metformin & $1 \mathrm{mM}, 5 \mathrm{mM}, 10 \mathrm{mM}$ & DMEM \\
\hline Nintedanib & $1 \mu M^{a}$ & DMSO \\
\hline Noggin & $200 \mathrm{ng} / \mathrm{mL}$ & Noggin reconstitution buffer \\
\hline Pirfenidone & $0.3 \mathrm{mg} / \mathrm{mL}^{\mathrm{a}}$ & Ethanol \\
\hline Selumetinib & $5 \mu \mathrm{M}^{\mathrm{a}}$ & DMSO \\
\hline rhTGF $\beta 1$ & $2 \mathrm{ng} / \mathrm{mL}$ & rhTGF $\beta 1$ reconstitution buffer \\
\hline rhBMP2 & $50 \mathrm{ng} / \mathrm{mL}^{\mathrm{a}}$ & rhBMP2 reconstitution buffer \\
\hline
\end{tabular}

aDosage was chosen based on a literature search and the dose showing highest efficacy was selected

prepared in two ways: lung specimens were cut into strips (length: $2-3 \mathrm{~cm}$, thickness: $3-5 \mathrm{~mm}$ ) and later chopped into $200-\mu \mathrm{m}$-thick slices using a Mcllwain Tissue Chopper (Campden Instruments Ltd.); or lung tissues were gently injected with $1.5 \%$ low-melting agarose (Roth) and cut using a vibratome (Thermo Fisher Scientific) into $400-\mu \mathrm{m}$-thick slices. Five to six PCLS were cultured in $5 \mathrm{~mL}$ of DMEM supplemented with $10 \% \mathrm{BCS}$ at $37^{\circ} \mathrm{C}$ and $5 \% \mathrm{CO}_{2}$ for 5 days. Cultures were treated with different agents at the beginning of the culture process.

siRNA transfection. siGENOME PRKAA1 siRNA (D-005027-01-0002), siGENOME PPARG siRNA (D-003436-03-0005), siGENOME BMP2 siRNA (D-01121902-0002), and the corresponding scrambled siRNA (siGENOME Non-Targeting siRNA \#2 (D-001210-02-05)) were obtained from Dharmacon. When cells reached $50-60 \%$ confluence in 6-well plates, they were transfected using lipofectamine RNAiMAX (Invitrogen) according to manufacturer's instructions (25 pmol per well, $7.5 \mu \mathrm{L}$ lipofectamine per well). The culture medium was replaced $24 \mathrm{~h}$ after transfection. Seventy-two hours after transfection, the culture medium was replaced by either fresh medium or fresh medium supplemented with $5 \mathrm{mM}$ metformin. After $72 \mathrm{~h}$, cells were harvested for protein or RNA extraction.

RNA extraction and quantitative real-time PCR. Total RNA extraction was performed using RNeasy mini or micro kits (Qiagen) and cDNA synthesis was carried out using Quantitect reverse transcription kit (Qiagen) according to manufacturer's instructions. Quantitative real-time PCR (qPCR) was performed using PowerUp SYBR green master mix (Applied Biosystems) and LightCycler 480 II machine (Roche Applied Science). Porphobilinogen deaminase (PBGD) was used as a reference gene. Data are presented as expression levels relative to $P B G D$ using the $2^{-\Delta \mathrm{CT}}$ method. Primer sequences are shown in Table 2.

Protein extraction and western blotting. Total protein lysates were prepared using RIPA buffer (Santa Cruz) according to manufacturer's instructions. Proteins were separated by sodium dodecyl sulfate-polyacrylamide gel electrophoresis (SDSPAGE) followed by blotting on polyvinylidene fluoride (PVDF) membranes (Thermo Fisher Scientific) using Trans-Blot SD semi-dry transfer cell (Bio-Rad Laboratories). Antibodies against AMPK (Abcam, 1:2500), PPAR $\gamma$ (H-100) (Santa Cruz, 1:1000), phospho-(Ser ${ }^{112}$ )-PPAR $\gamma$ (Merck Millipore, 1:500), p44/42 mitogenactivated protein kinase (MAPK, a.k.a. extracellular signal-regulated kinases 1 and 2, ERK1/2) (Cell Signaling, 1:1000), phospho-p44/42 MAPK [(ERK1) (Tyr204)/ (ERK2) (Tyr187) (D1H6G)] (Cell Signaling, 1:1000), and cleaved poly(ADPribose) polymerase 1 (PARP1) (Abcam, 1:2000) were used overnight at $4{ }^{\circ} \mathrm{C}$. Betaactin (ACTB) was used as a loading control (Biolegend, 1:2500). HRP-conjugated anti-rabbit IgG (Promega, 1:5000), HRP-conjugated anti-mouse IgG $(\mathrm{H}+\mathrm{L})$ (Promega, 1:5000), and HRP-conjugated anti-rat IgG (Biolegend, 1:5000) were used as secondary antibodies for $1 \mathrm{~h}$ at room temperature (RT). Subsequently, membranes were covered with AceGlow chemiluminescence substrate (Peqlab) and imaged immediately using ChemiDoc XRS + (Bio-Rad Laboratories).

Staining for lipid-droplet accumulation. For time-lapse imaging, live cells were treated with different agents and HCS LipidTOX red neutral lipid dye (Invitrogen, 1:200) was immediately added. Cells were then placed in the incubation chamber $\left(37^{\circ} \mathrm{C}\right.$ and $5 \% \mathrm{CO}_{2}$ ) of DMI6000 B live imaging microscope (Leica). Images were 
Table 2 Primer sequences for $\mathrm{qPCR}$

Gene name

BMP2

COL1A1

PBGD

PLIN2

PPARg
Forward primer

CAGACCACCGGTTGGAGA

ATGTTCAGCTTTGTGGACCTC

TGTCTGGTAACGGCAATGCG

TCAGCTCCATTCTACTGTTCACC

TTGCTGTCATTATTCTCAGTGGA
Reverse primer

CCACTCGTTTCTGGTAGTTCTTC

CTGTACGCAGGTGATTGGTG

CCCACGCGAATCACTCTCAT

CCTGAATTTTCTGATTGGCAC

GAGGACTCAGGGTGGTTCAG acquired every $1 \mathrm{~h}$. In other cases, cells were fixed using $2 \%$ paraformaldehyde (PFA, Roth) for $20 \mathrm{~min}$ followed by washing with PBS (Gibco). A mixture of diluted LipidTOX (1:200) and Hoechst (1:5000) in PBS was then used to stain fixed cells. Subsequently, slides were mounted using ProLong Gold Antifade Reagent (Molecular Probes).

Hematoxylin and eosin staining. Human-derived PCLS or mouse lung tissues were fixed using $4 \%$ PFA followed by embedding in paraffin. Paraffin blocks were sectioned into 5 - $\mu \mathrm{m}$-thick slices and placed on glass slides. Following deparaffinization, lung sections were stained with hematoxylin (Roth) for $2 \mathrm{~min}$, washed with running tap water for $10 \mathrm{~min}$ and then stained with eosin (Thermo Fisher Scientific) for $2 \mathrm{~min}$.

Masson's trichrome staining and fibrosis quantification. Collagen staining was performed using Masson's trichrome staining kit according to the protocol recommended by the manufacturer (Thermo Fisher Scientific). Fibrosis was assessed by semi-automated quantification using VIS Image Analysis Software (Visiopharm). In brief, the algorithm calculates the percentage of the area covered by collagen fibers relative to the area covered by lung tissue (excluding airways and airspaces).

Total collagen assay. In order to assess total collagen levels, $10 \mathrm{mg}$ of each tissue sample was subjected to the total collagen assay kit according to manufacturer's instructions (Biovision).

Immunofluorescence. Following deparaffinization, slides underwent antigen retrieval using citrate buffer (Vector Laboratories) for $15 \mathrm{~min}$ followed by cooling on ice for $20 \mathrm{~min}$. Slides were then blocked with $3 \%$ bovine serum albumin (BSA, Jackson Immunoresearch Laboratories) in PBS for $1 \mathrm{~h}$ at RT. Anti-collagen $1 \mathrm{~A} 1$ (anti-COL1A1) antibodies (Rockland, 1:200) and goat anti-rabbit antibodies (Life Technologies, 1:500) were used for immunofluorescence. Slides were finally mounted with ProLong Gold Antifade Reagent containing DAPI (Molecular Probes).

Human PCLS were fixed in 4\% PFA for $2 \mathrm{~h}$ and stored in PBS containing $0.02 \%$ sodium azide. Fixed PCLS were blocked with $3 \%$ BSA in PBS for $2 \mathrm{~h}$ at RT, stained with anti-COL1A1 antibodies (Rockland, 1:200) overnight at $4{ }^{\circ} \mathrm{C}$ and washed with PBS (three times, $30 \mathrm{~min}$ each). A mixture of Alexa Fluor 488-conjugated antirabbit antibodies (Invitrogen, 1:200), HCS LipidTOX red neutral lipid dye (Invitrogen, 1:200) and Hoechst (1:5000) was added for $3 \mathrm{~h}$ at RT. After washing, PCLS were placed in glass-bottomed 4-well micro slides (Ibidi) containing PBS and imaged by confocal microscopy (Leica TCS SP5).

For ACTA2 immunostaining following PFA fixation, samples were blocked with $3 \%$ BSA in PBS supplemented with $0.4 \%$ Triton-X (Sigma-Aldrich) for $1 \mathrm{~h}$ at RT and then stained with FITC-conjugated mouse monoclonal anti-ACTA2 antibodies (Sigma-Aldrich,1:200) overnight at $4^{\circ} \mathrm{C}$. Slides were finally mounted with ProLong Gold Antifade Reagent containing DAPI (Molecular Probes).

Flow cytometry. Cultured fibroblasts were washed with PBS and resuspended in PBS containing LipidTOX (1:200). Following incubation for $30 \mathrm{~min}$, cells were subjected to flow cytometry using Accuri C6 (BD Biosciences). Cultured PCLS and/ or finely minced mouse lung tissues underwent digestion with $0.5 \%$ collagenase (Gibco) in PBS for $45 \mathrm{~min}$ at $37^{\circ} \mathrm{C}$ while rotating. Cell suspensions were then aspirated through $18,20,24 \mathrm{G}$ needles and passed through 70 - and $40-\mu \mathrm{m}$ cell strainers (Greiner Bio-One). Cells were pelleted, resuspended in PBS and stained with anti-CD45 (Biolegend, 1:100), CD31 (Biolegend, 1:100) and CD326 (EpCAM, Biolegend, 1:50) antibodies, as well as LipidTOX (1:200). Stained cell suspensions were then analyzed using LSRFortessa (BD Biosciences). Data were analyzed using FlowJo software (FlowJo, LLC).

Gene expression microarrays. Total RNA Cy5-labeling was carried out using the LIRAK kit (Agilent Technologies) according to manufacturer's instructions. Per reaction, 200-ng of total RNA was used. Cy-labeled aRNA was hybridized overnight to $8 \times 60 \mathrm{~K} 60 \mathrm{mer}$ oligonucleotide-spotted microarray slides (Agilent Technologies, design ID 028005). Hybridization and subsequent washing and drying of the slides were performed following the Agilent hybridization protocol. Dried slides were scanned at $2 \mu \mathrm{m} /$ pixel resolution using the InnoScan is900 (Innopsys). Image analysis was performed using Mapix 6.5.0 software and calculated values for all spots were saved as GenePix results files. Stored data were evaluated using the R software and the limma package from BioConductor. Log mean spot signals were taken for further analysis. Data were quantile-normalized before averaging. Genes were ranked for differential expression using a moderated $t$-statistic. Pathway analyses were done using gene set tests on the ranks of $t$-statistics.

Kinase activity assay. Kinase activity of metformin- or vehicle-treated IPF fibroblasts ( $n=3$ per group) was analyzed by the PamStation (PamGene International BV) that uses a methodology that allows robust analysis of the activity of tyrosine as well as serine/threonine kinases in cells and tissues ${ }^{50-53}$. Hereby, active kinases phosphorylate their distinct peptide substrates presented on a peptide array chip. Phosphorylated peptides are recognized by phospho-specific FITC-labeled antibodies and detection, performed in multiple cycles at different exposure times, is monitored by a CCD camera. Software-based image analysis integrates the signals obtained within the time course of the incubation of the kinase lysate on the chip into one single value for each peptide for each sample (exposure time scaling). Log transformation of processed signals allows easier graphical presentation of the raw data. Thereby, data with significant differences in intensity are visualized on the log-transformed $y$-axis in a heatmap that shows the degree of phosphorylation for each peptide.

For protein isolation including active kinases, IPF fibroblasts were washed with $5 \mathrm{~mL}$ ice-cold PBS and scraped from the dishes in $100 \mu \mathrm{L}$ of M-PER lysis buffer (Thermo Fisher Scientific) containing protease and phosphatase inhibitor cocktails (Pierce). The lysate was incubated for $1 \mathrm{~h}$ at $4{ }^{\circ} \mathrm{C}$ with constant agitation followed by centrifugation at $16,000 \times g$ for $15 \mathrm{~min}$ at $4^{\circ} \mathrm{C}$. The supernatant was immediately flash-frozen in liquid nitrogen and stored at $-80^{\circ} \mathrm{C}$. Protein concentration was determined using a bicinchoninic acid (BCA) protein assay kit (Thermo Fisher Scientific) according to manufacturer's instructions.

For tyrosine kinase activity detection, $10 \mu \mathrm{g}$ of protein lysate was dispensed onto an array of the PamChip PTK (phospho-tyrosine kinase) dissolved in protein kinase buffer and additives (proprietary information) including $1 \%$ BSA, $10 \mathrm{mM}$ DTT, $0.6 \mu \mathrm{L}$ FITC-conjugated antibodies and $400 \mu \mathrm{M}$ ATP in a final volume of $40 \mu \mathrm{L}$ (assay master mix). A total of $1 \mu \mathrm{g}$ of protein lysate was used for serine/ threonine kinase activity detection on an array of the PamChip STK (serine/ threonine kinase) with protein kinase buffer (proprietary information) supplied with $1 \%$ BSA, $0.46 \mu \mathrm{l}$ primary STK antibody mix and $400 \mu \mathrm{M}$ ATP (sample mix) After an initial incubation time, secondary FITC-labeled antibodies $(0.4 \mu \mathrm{L})$ were added. The mixture was dissolved in antibody buffer (proprietary information) and water in a final volume of $30 \mu \mathrm{L}$ (detection mix).

Upstream kinase prediction on the basis of the different phosphorylation pattern in metformin- and vehicle-treated IPF fibroblasts was conducted using the Bionavigator software v.6.3.67.0 (PamGene International).

Statistical analyses. Statistical analyses and graph assembly were carried out using GraphPad Prism 6 (GraphPad Prism Software). To assess the normal distribution of data sets, D'Agostino-Pearson normality test was applied. In case of normal distribution, Student's $t$-test (unpaired, two-tailed) was utilized to compare the means of two groups, while one-way ANOVA (with post hoc analysis) was used to compare the means of three or more groups. In cases where the data were not normally distributed or the normality test could not be applied, respective nonparametric tests were applied (Mann-Whitney test to compare the means of two groups and Kruskal-Wallis test to compare the means of three or more groups). Data are presented as mean \pm standard error of mean (s.e.m.). The number of biological samples $(n)$ for each group and the utilized statistical tests are stated in the corresponding figure legends. Differences in means were considered statistically significant if $P<0.05$.

Reporting summary. Further information on research design is available in the Nature Research Reporting Summary linked to this article. 


\section{Data availability}

The data sets generated during and/or analyzed during the current study are available in Gene Expression Omnibus (GEO) under the accession number GSE131133 .

Received: 16 January 2019 Accepted: 3 June 2019

Published online: 05 July 2019

\section{References}

1. Sgalla, G., Biffi, A. \& Richeldi, L. Idiopathic pulmonary fibrosis: diagnosis, epidemiology and natural history. Respirology 21, 427-437 (2016).

2. Todd, N. W., Luzina, I. G. \& Atamas, S. P. Molecular and cellular mechanisms of pulmonary fibrosis. Fibrogenes. Tissue Repair 5, 11 (2012).

3. Günther, A. et al. Unravelling the progressive pathophysiology of idiopathic pulmonary fibrosis. Eur. Respir. Rev. 21, 152-160 (2012).

4. El Agha, E. et al. Mesenchymal stem cells in fibrotic disease. Cell Stem Cell 21, 166-177 (2017).

5. Rock, J. R. et al. Multiple stromal populations contribute to pulmonary fibrosis without evidence for epithelial to mesenchymal transition. Proc. Natl Acad. Sci. USA. 108, E1475-E1483 (2011).

6. Xie, T. et al. Transcription factor TBX4 regulates myofibroblast accumulation and lung fibrosis. J. Clin. Invest. 126, 3626 (2016).

7. Kramann, R. et al. Perivascular Gli1+ progenitors are key contributors to injury-induced organ fibrosis. Cell Stem Cell 16, 51-66 (2015).

8. El Agha, E. et al. Two-way conversion between lipogenic and myogenic fibroblastic phenotypes marks the progression and resolution of lung fibrosis. Cell Stem Cell 20, 261-273.e3 (2017).

9. Genovese, T. et al. Effect of rosiglitazone and 15-deoxy-Delta12,14prostaglandin $\mathrm{J} 2$ on bleomycin-induced lung injury. Eur. Respir. J. J. Eur. Soc. Clin. Respir. Physiol. 25, 225-234 (2005).

10. Burgess, H. A. et al. PPARgamma agonists inhibit TGF-beta induced pulmonary myofibroblast differentiation and collagen production: implications for therapy of lung fibrosis. Am. J. Physiol. Lung Cell. Mol. Physiol. 288, L1146-L1153 (2005).

11. Mirakaj, V. et al. Rosiglitazone dampens pulmonary inflammation in a porcine model of acute lung injury. Inflammation 37, 1102-1110 (2014).

12. Lyon, C. M. et al. Rosiglitazone prevents the progression of preinvasive lung cancer in a murine model. Carcinogenesis 30, 2095-2099 (2009).

13. Pouwels, K. B. \& van Grootheest, K. The rosiglitazone decision process at FDA and EMA. What should we learn? Int J. Risk Saf. Med. 24, 73-80 (2012).

14. Zhao, Y. D. et al. Metabolic heterogeneity of idiopathic pulmonary fibrosis: a metabolomic study. BMJ Open Respir. Res. 4, e000183 (2017).

15. Yan, F. et al. Identification of the lipid biomarkers from plasma in idiopathic pulmonary fibrosis by Lipidomics. BMC Pulm. Med. 17, 174 (2017).

16. García-Sancho Figueroa, M. C. et al. Risk factors for idiopathic pulmonary fibrosis in a Mexican population. A case-control study. Respir. Med. 104, 305-309 (2010).

17. Li, C., Xue, Y., Xi, Y. R. \& Xie, K. Progress in the application and mechanism of metformin in treating non-small cell lung cancer. Oncol. Lett. 13, 2873-2880 (2017).

18. Yu, T. et al. Metformin inhibits SUV39H1-mediated migration of prostate cancer cells. Oncogenesis 6, e324 (2017).

19. Castilla-Guerra, L., Fernandez-Moreno, M. D. C., Leon-Jimenez, D. \& Carmona-Nimo, E. Antidiabetic drugs and stroke risk. Curr. Evid. Eur. J. Intern. Med. 48, 1-5 (2018).

20. Sato, N. et al. Metformin attenuates lung fibrosis development via NOX4 suppression. Respir. Res. 17, 107 (2016).

21. Rangarajan, $\mathrm{S}$. et al. Metformin reverses established lung fibrosis in a bleomycin model. Nat. Med. 24, 1121-1127 (2018).

22. Westergren-Thorsson, G. et al. Increased deposition of glycosaminoglycans and altered structure of heparan sulfate in idiopathic pulmonary fibrosis. Int. J. Biochem. Cell Biol. 83, 27-38 (2017).

23. Lu, J., Auduong, L., White, E. S. \& Yue, X. Upregulation of heparan sulfate 6O-sulfation in idiopathic pulmonary fibrosis. Am. J. Respir. Cell Mol. Biol. 50, 130820094406000 (2013).

24. Kulkarni, T., O’Reilly, P., Antony, V. B., Gaggar, A. \& Thannickal, V. J. Matrix remodeling in pulmonary fibrosis and emphysema. Am. J. Respir. Cell Mol. Biol. 54, 751-760 (2016).

25. Uhl, F. E. et al. Preclinical validation and imaging of Wnt-induced repair in human 3D lung tissue cultures. Eur. Respir. J. 46, 1150-1166 (2015).

26. Hansmann, G. et al. An antiproliferative BMP-2/PPARgamma/apoE axis in human and murine SMCs and its role in pulmonary hypertension. J. Clin. Invest. 118, 1846-1857 (2008).

27. Calvier, L. et al. PPAR $\gamma$ links BMP2 and TGF $\beta 1$ pathways in vascular smooth muscle cells, regulating cell proliferation and glucose metabolism. Cell Metab. 25, 1118-1134.e7 (2017).
28. Zhang, B. et al. Insulin- and mitogen-activated protein kinase-mediated phosphorylation and activation of peroxisome proliferator-activated receptor \%. J. Biol. Chem. 271, 31771-31774 (1996).

29. Compe, E. et al. Dysregulation of the peroxisome proliferator-activated receptor target genes by XPD mutations. Mol. Cell. Biol. 25, 6065-6076 (2005)

30. Iankova, I. et al. Peroxisome proliferator-activated receptor $\gamma$ recruits the positive transcription elongation factor $\mathrm{b}$ complex to activate transcription and promote adipogenesis. Mol. Endocrinol. 20, 1494-1505 (2006).

31. Madala, S. K. et al. MEK-ERK pathway modulation ameliorates pulmonary fibrosis associated with epidermal growth factor receptor activation. Am. J. Respir. Cell Mol. Biol. 46, 380-388 (2012).

32. Hosooka, T. et al. Dok1 mediates high-fat diet-induced adipocyte hypertrophy and obesity through modulation of PPAR $-\gamma$ phosphorylation. Nat. Med. 14, 188-193 (2008).

33. Leonard, C. E. et al. Comparative risk of serious hypoglycemia with oral antidiabetic monotherapy: a retrospective cohort study. Pharmacoepidemiol Drug Saf. 27, 9-18 (2018).

34. Siersbæk, R., Nielsen, R. \& Mandrup, S. PPAR $\gamma$ in adipocyte differentiation and metabolism-Novel insights from genome-wide studies. FEBS Lett. 584, 3242-3249 (2010).

35. Malur, A. et al. Deletion of PPAR $\gamma$ in Alveolar macrophages is associated with a Th-1 pulmonary inflammatory response. J. Immunol. 182, 5816 (2009).

36. Sun, H. et al. Osteoblast-targeted suppression of PPAR $\gamma$ increases osteogenesis through activation of mTOR signaling. Stem Cells 31, https://doi.org/10.1002/ stem.1455 (2013).

37. Lodhi, I. J. \& Semenkovich, C. F. Peroxisomes: a nexus for lipid metabolism and cellular signaling. Cell Metab. 19, 380-392 (2014).

38. Varisco, B. M., Ambalavanan, N., Whitsett, J. A. \& Hagood, J. S. Thy-1 signals through PPAR $\gamma$ to promote lipofibroblast differentiation in the developing lung. Am. J. Respir. Cell Mol. Biol. 46, 765-772 (2012).

39. Rehan, V. K. \& Torday, J. S. PPAR $\gamma$ signaling mediates the evolution, development, homeostasis, and repair of the lung. PPAR Res. 2012, 289867 (2012).

40. Chen, S. C. et al. Metformin suppresses adipogenesis through both AMPactivated protein kinase (AMPK)-dependent and AMPK-independent mechanisms. Mol. Cell. Endocrinol. 440, 57-68 (2017).

41. Alexandre, K. B., Smit, A. M., Gray, I. P. \& Crowther, N. J. Metformin inhibits intracellular lipid accumulation in the murine pre-adipocyte cell line, 3T3-L1. Diabetes, Obes. Metab. 10, 688-690 (2008).

42. Viollet, B. et al. Cellular and molecular mechanisms of metformin: an overview. Clin. Sci. (Lond.). 122, 253-270 (2012).

43. González-Barroso, M. M. et al. Fatty acids revert the inhibition of respiration caused by the antidiabetic drug metformin to facilitate their mitochondrial $\beta$ oxidation. Biochim. Biophys. Acta-Bioenerg. 1817, 1768-1775 (2012)

44. Phielix, E., Szendroedi, J. \& Roden, M. The role of metformin and thiazolidinediones in the regulation of hepatic glucose metabolism and its clinical impact. Trends Pharm. Sci. 32, 607-616 (2011).

45. Spagnolo, P. et al. Metformin does not affect clinically relevant outcomes in patients with idiopathic pulmonary fibrosis. Respiration 96, 314-322 (2018).

46. Tzouvelekis, A., Tzilas, V., Dassiou, M. \& Bouros, D. Metformin in idiopathic pulmonary fibrosis "seeking the holy-grail through drug-repositioning". Respiration 96, 305-307 (2018).

47. Guenther, A. et al. The European IPF registry (eurIPFreg): baseline characteristics and survival of patients with idiopathic pulmonary fibrosis. Respir. Res. 19, 141 (2018).

48. Wendling, O., Bornert, J.-M., Chambon, P. \& Metzger, D. Efficient temporally-controlled targeted mutagenesis in smooth muscle cells of the adult mouse. Genesis 47, 14-18 (2009).

49. Madisen, L. et al. A robust and high-throughput Cre reporting and characterization system for the whole mouse brain. Nat. Neurosci. 13, 133-140 (2010).

50. Piersma, S. R., Labots, M., Verheul, H. M. W. \& Jiménez, C. R. Strategies for kinome profiling in cancer and potential clinical applications: chemical proteomics and array-based methods. Anal. Bioanal. Chem. 397, 3163-3171 (2010).

51. Arsenault, R., Griebel, P. \& Napper, S. Peptide arrays for kinome analysis: new opportunities and remaining challenges. Proteomics 11, 4595-4609 (2011).

52. Anderson, J. C. et al. High throughput kinomic profiling of human clear cell renal cell carcinoma identifies kinase activity dependent molecular subtypes. PLOS ONE 10, e0139267 (2015).

53. Anderson, J. C. et al. Correction: kinomic profiling of electromagnetic navigational bronchoscopy specimens: a new approach for personalized medicine. PLoS ONE 11, e0161986 (2016).

\section{Acknowledgements}

E.E.A. was funded by a start-up grant from the Excellence Cluster Cardio-Pulmonary System (ECCPS). E.E.A. also acknowledges the support of the Cardio-Pulmonary Institute (CPI; EXC 2026, Project ID: 390649896), University Hospital Giessen and 
Marburg (UKGM) and the German Center for Lung Research (DZL). S.B. was supported by grants from the Deutsche Forschungsgemeinschaft (DFG; BE4443/1-1, BE4443/4-1, BE4443/6-1, KFO309 P7 and Project number 268555672-SFB1213, projects A02 and A04), Landes-Offensive zur Entwicklung Wissenschaftlich-Ökonomischer Exzellenz (LOEWE), UKGM, Universities of Giessen and Marburg Lung Center (UGMLC), DZL, and COST (BM1201). J.S.Z. was funded through a start-up package from Wenzhou Medical University and the National Natural Science Foundation of China (grant number 81472601). S.H. was supported by the UKGM (FOKOOPV), the DZL and grants from the DFG (KFO309 P2/8; SFB1021 C05, SFB TR84 B9).

\section{Author contributions}

Conceived the study: R.W., W.S., A.G., X.L., S.B., and E.E.A. Acquired and analyzed the data: V.K., R.W., V.B., A.V., X.C., A.M., A.W., J.W., S.B., and E.E.A. Contributed to methodology: J.S.Z., B.M., G.K., S.H., R.T.S., A.G., S.B., and E.E.A. Drafted the manuscript: V.K. and E.E.A. Edited the manuscript: R.W., V.B., A.V., J.W., G.K., S.H., W.S., A.G., S.B. and E.E.A.

\section{Additional information}

Supplementary Information accompanies this paper at https://doi.org/10.1038/s41467019-10839-0.

Competing interests: The authors declare no competing interests.
Reprints and permission information is available online at http://npg.nature.com/ reprintsandpermissions/

Peer review information: Nature Communications thanks the anonymous reviewers for their contribution to the peer review of this work.

Publisher's note: Springer Nature remains neutral with regard to jurisdictional claims in published maps and institutional affiliations.

(c)

Open Access This article is licensed under a Creative Commons Attribution 4.0 International License, which permits use, sharing, adaptation, distribution and reproduction in any medium or format, as long as you give appropriate credit to the original author(s) and the source, provide a link to the Creative Commons license, and indicate if changes were made. The images or other third party material in this article are included in the article's Creative Commons license, unless indicated otherwise in a credit line to the material. If material is not included in the article's Creative Commons license and your intended use is not permitted by statutory regulation or exceeds the permitted use, you will need to obtain permission directly from the copyright holder. To view a copy of this license, visit http://creativecommons.org/ licenses/by/4.0/.

(C) The Author(s) 2019 